JOURNAL OF

SYMPLECTIC GEOMETRY

Volume 9, Number 1, 45-82, 2011

\title{
SYMPLECTIC MAPPING CLASS GROUPS OF SOME STEIN AND RATIONAL SURFACES
}

\author{
JONATHAN DAVID EvANS
}

In this paper we compute the homotopy groups of the symplectomorphism groups of the three-, four- and five-point blow-ups of the projective plane (considered as monotone symplectic Del Pezzo surfaces). Along the way, we need to compute the homotopy groups of the compactly supported symplectomorphism groups of the cotangent bundle of $\mathbb{R}^{2}$ and of $\mathbb{C}^{*} \times \mathbb{C}$. We also make progress in the case of the $A_{n}$-Milnor fibres: here we can show that the (compactly supported) Hamiltonian group is contractible and that the symplectic mapping class group embeds in the braid group on $n$-strands.

\section{Contents}

1. Introduction $\quad 46$

1.1. Review 46

1.2. New results $\quad 47$

1.3. Comments $\quad 48$

1.4. Philosophy 49

1.5. Overview $\quad 49$

2. Stein manifolds $\quad 50$

2.1. Stein manifolds and Liouville flows 50

2.2. Symplectomorphism groups $\quad 52$

3. $\operatorname{Symp}_{\mathrm{c}}\left(T^{*} \mathbb{R} \mathbb{P}^{2}\right)$

4. Groups associated to configurations of spheres $\quad 56$

4.1. Gauge transformations $\quad 56$

4.2. Surface symplectomorphisms $\quad 57$

4.3. Configurations of spheres $\quad 58$

4.4. Banyaga's isotopy extension theorem 59

5. $\operatorname{Symp}_{\mathbf{c}}\left(\mathbb{C}^{*} \times \mathbb{C}\right) \quad 59$ 
5.1. Proof of Theorem 1.6

5.2. Proof of Proposition 5.1

5.2.2. Structures making a configuration holomorphic

5.2.3. Gromov's theory of pseudoholomorphic curves 64 5.2.4. Proof of Proposition 5.1

6. Del Pezzo surfaces

6.1. Results

6.3. The case $\mathbb{D}_{3}$

6.4. The case $\mathbb{D}_{4}$

6.5. The case $\mathbb{D}_{5}$

7. The $A_{n}$-Milnor fibres $\quad 72$

7.1. Compactification $\quad 72$

7.2. Proof of Theorem $1.4 \quad 74$

8. Appendix A. Structures making a configuration holomorphic

\section{Introduction}

A central object in symplectic topology is the group $\operatorname{Symp}(X, \omega)$ of (compactly supported) diffeomorphisms of a symplectic manifold $X$ which preserve the symplectic form $\omega$. This paper studies the weak homotopy type of $\operatorname{Symp}(X, \omega)$ for some simple symplectic four-manifolds. We begin by reviewing what is known about the topology of symplectomorphism groups.

1.1. Review. Gromov [Gro85] gave a beautiful proof of the following theorem:

Theorem 1.1. The symplectomorphism group of $S^{2} \times S^{2}$ with its product symplectic form $\omega \oplus \omega$ deformation retracts onto $\mathrm{SO}(3) \times \mathrm{SO}(3) \rtimes \mathbb{Z} / 2$.

This is just one corollary of his theory of pseudoholomorphic curves in symplectic manifolds. The tractability of $\operatorname{Symp}\left(S^{2} \times S^{2}\right)$ derives from the following existence theorem for pseudoholomorphic foliations:

Theorem 1.2. If $J$ is an almost complex structure on $X=S^{2} \times S^{2}$ which is compatible with the product symplectic form $\omega$ then there exist $J$-holomorphic foliations $\mathcal{F}_{1}$ and $\mathcal{F}_{2}$ of $X$ whose leaves are spheres in the homology classes $S^{2} \times\{\mathrm{pt}\}$ and $\{\mathrm{pt}\} \times S^{2}$, respectively. 
Since $J$-holomorphic curves intersect positively, a leaf of $\mathcal{F}_{1}$ and a leaf of $\mathcal{F}_{2}$ intersect transversely in a single point. This allows one to construct diffeomorphisms conjugating the foliation pairs coming from different almost complex structures. Modifications of these arguments allow one to deduce that:

- $\operatorname{Symp}\left(\mathbb{C P}^{2}, \omega\right)$ is homotopy equivalent to $\mathbb{P U}(3)$, where $\omega$ is the Fubini-Study form (due to Gromov [Gro85]),

- $\operatorname{Symp}\left(\mathbb{D}_{1}, \omega\right)$ is homotopy equivalent to $U(2)$, where $\mathbb{D}_{1}$ is the onepoint blow-up of $\mathbb{C P}^{2}$ and $\omega$ is the anticanonical Kähler form (due to Gromov [Gro85]),

- $\operatorname{Symp}\left(\mathbb{D}_{2}, \omega\right)$ is weakly homotopy equivalent to $T^{2} \rtimes \mathbb{Z} / 2$, where $\mathbb{D}_{2}$ is the two-point blow-up of $\mathbb{C P}^{2}$ and $\omega$ is the anticanonical Kähler form (due to Lalonde-Pinsonnault [LP04]),

- $\operatorname{Symp}_{\mathrm{c}}\left(\mathbb{C}^{2}\right)$ is contractible, where $\omega$ is the product symplectic form (due to Gromov [Gro85]),

- $\operatorname{Symp}_{\mathrm{c}}\left(T^{*} S^{2}\right)$ is weakly homotopy equivalent to $\mathbb{Z}$, where $\omega$ is the canonical symplectic form (by argument due to Seidel, see [Sei98]).

Here Symp $_{c}$ denotes the group of compactly supported symplectomorphisms. These arguments work because $\mathbb{C}^{2}$ and $T^{*} S^{2}$ embed into $S^{2} \times S^{2}$ and inherit foliations by pseudoholomorphic discs from the foliations of Theorem 1.2 .

One might hope that if one could calculate $\left\{\pi_{i}\left(\operatorname{Symp}_{\mathrm{c}}(A)\right)\right\}_{i \in \mathbb{N}}$ for some other affine varieties $A$ then one might use Gromov's theory of pseudoholomorphic curves in symplectic four-manifolds to deduce something about the topology of $\operatorname{Symp}(X)$ where $X$ is a projective variety containing $A$ as a Zariski open set. This paper accomplishes this in a small number of cases.

Note that there are also some results on weak contractibility of Symp $_{c}$ for symplectic disc bundles over $S^{2}$, from the work of Richard Hind [Hin03] and on $\operatorname{Symp}_{c}\left(\mathbb{C}^{*} \times \mathbb{C}^{*}\right)$ from the work of Chris Wendl [Wen08]. These results are based on arguments from symplectic field theory. There is also a lot of work about the topology of $\operatorname{Symp}\left(S^{2} \times S^{2}, \omega\right)$ where $\omega$ is a nonmonotone symplectic form (e.g., see $[\mathbf{A b r} \mathbf{9 8}])$. In this paper, we are almost exclusively concerned with monotone symplectic forms (except in the final section), so we do not review this literature. It should be noted, however, that the techniques of [A $\mathbf{A b r 9 8}]$ have heavily inspired the techniques of this paper and this will be immediately evident to readers who are familiar with that work.

1.2. New results. The aim of this paper is to add to the list of symplectic four-manifolds whose symplectomorphism group we understand (up to weak homotopy equivalence). In particular, we prove the following theorems:

Theorem 1.3. Let $\mathbb{D}_{n}$ denote the blow-up of $\mathbb{C P}^{2}$ at $n \leq 5$ points in general position and let $\omega$ denote its anticanonical Kähler form. In each case let 
$\operatorname{Symp}_{0}\left(\mathbb{D}_{n}\right)$ denote the group of symplectomorphisms of $\left(\mathbb{D}_{n}, \omega\right)$ which act trivially on $H_{*}\left(\mathbb{D}_{n}, \mathbb{Z}\right)$.

- $\operatorname{Symp}_{0}\left(\mathbb{D}_{3}\right)$ is weakly homotopy equivalent to $T^{2}$,

- $\operatorname{Symp}_{0}\left(\mathbb{D}_{4}\right)$ is weakly contractible and

- $\operatorname{Symp}_{0}\left(\mathbb{D}_{5}\right)$ is weakly homotopy equivalent to $\operatorname{Diff}^{+}\left(S^{2}, 5\right)$, the group of orientation-preserving diffeomorphisms of $S^{2}$ preserving five points.

The author has since learned that these results on monotone symplectic Del Pezzo surfaces have also been obtained independently by Martin Pinsonnault [Pin].

Theorem 1.4. Let $W$ be the $A_{n}$-Milnor fibre, the affine variety given by the equation

$$
x^{2}+y^{2}+z^{n}=1,
$$

and let $\omega$ be the Kähler form on $W$ induced from the ambient $\mathbb{C}^{3}$. Then the group of compactly supported symplectomorphisms of $(W, \omega)$ is weakly homotopy equivalent to its group of components. This group of components injects homomorphically into the braid group $\mathrm{Br}_{n}$ of $n$-strands on the disc.

En route to proving these theorems, we prove and use:

Theorem 1.5. Let $\omega$ be the canonical symplectic form on the cotangent bundle of $\mathbb{R P}^{2}$. The group of compactly supported symplectomorphisms $\operatorname{Symp}_{\mathrm{c}}\left(T^{*} \mathbb{R} \mathbb{P}^{2}\right)$ is weakly homotopy equivalent to $\mathbb{Z}$.

Theorem 1.6. Let $\omega$ be the product symplectic form on $\mathbb{C}^{*} \times \mathbb{C}$. The group of compactly supported symplectomorphisms $\operatorname{Symp}_{\mathrm{c}}\left(\mathbb{C}^{*} \times \mathbb{C}\right)$ is weakly contractible.

1.3. Comments. Theorem 1.4 is not as strong as we would like: the injection is probably also a surjection. I have been unable to prove this for technical reasons. However, we can still deduce something more about the symplectic mapping class group $\pi_{0}\left(\operatorname{Symp}_{\mathrm{c}}(W)\right)$ in the case $n \geq 4$. Khovanov and Seidel demonstrated [KS01] using Fukaya categories that the braid group $\mathrm{Br}_{n}$ always injects into $\pi_{0}\left(\operatorname{Symp}_{\mathrm{c}}(W)\right)$. The composition of their injection with ours is therefore a homomorphic injection $K: \mathrm{Br}_{n} \rightarrow \mathrm{Br}_{n}$. While the braid group is not co-Hopfian, it is known [BM06] that, when $n \geq 4$, all such injections are of the form

$$
\sigma_{i} \mapsto z^{\ell}\left(h^{-1} \sigma_{i} h\right)^{ \pm 1},
$$

where $\sigma_{i}$ are the usual generators, $h$ is a homeomorphism of the marked disc, $z \in Z\left(\mathrm{Br}_{n}\right)$ is a full-twist and $\ell \in \mathbb{Z}$. In particular, we know that the subgroup $K\left(\mathrm{Br}_{n}\right)$ is of finite index and that all intermediate subgroups between $K\left(\mathrm{Br}_{n}\right)$ and $\mathrm{Br}_{n}$ are isomorphic to $\mathrm{Br}_{n}$, in particular the group $\pi_{0}\left(\operatorname{Symp}_{\mathrm{c}}(W)\right)$ is abstractly isomorphic to $\mathrm{Br}_{n}$. 
Seidel [Sei08] has also obtained results in the direction of Theorem 1.3 , specifically exhibiting a subgroup of $\pi_{0}\left(\operatorname{Symp}_{0}\left(\mathbb{D}_{5}\right)\right)$ isomorphic to $\pi_{0}\left(\operatorname{Diff}^{+}\left(S^{2}, 5\right)\right)$. These results were the starting point for this paper and will be reviewed in Section 6.1.

Note that the weak homotopy equivalences proved in the theorems actually suffice to prove homotopy equivalence since, by McDuff and Salamon [MS04, Remark 9.5.6], these topological groups have the homotopy type of a countable CW complex.

1.4. Philosophy. The methods of this paper are very classical, along the lines of $[\mathbf{A b r 9 8 , ~ L P 0 4}]$; the only "hard" input is from Gromov's theory of pseudoholomorphic curves. In each case we will identify a divisor in our symplectic manifold (or in a compactification of it). The divisor will only have spherical components. The idea is to relate the topology of the symplectomorphism group to the topology of the "symplectomorphism group" of the divisor, i.e., the group of symplectomorphisms of the components that fix intersection points between different components. For instance, as observed by Seidel, the divisor we use in $\mathbb{D}_{5}$ contains a component with five intersection points, leading to the pure braid group on five strands. The difficulty is in working out the precise relationship of this to the symplectomorphism group and showing there is no topology invisible to this divisor. This involves examining a number of long exact sequences of homotopy groups coming from fibrations and understanding the topology of the compactly supported symplectomorphism group of the complement of the divisor.

One might ask why we stopped at $\mathbb{D}_{5}$. The reason is that the complement of a conic in $\mathbb{C P}^{2}$ (symplectically $T^{*} \mathbb{R P}^{2}$ ) occurs naturally as a Zariski open subset here. $\mathbb{D}_{6}$ is a cubic surface, so one might hope to understand its symplectomorphism group if one could understand symplectomorphisms of the affine cubic surface. However, this does not admit a foliation by rational holomorphic curves, which is ultimately what allows us to understand $\operatorname{Symp}_{\mathrm{c}}\left(T^{*} \mathbb{R} \mathbb{P}^{2}\right)$.

1.5. Overview. Section 2 reviews the properties of non-compact symplectic manifolds and their (compactly supported) symplectomorphism groups relevant for later arguments. The aim of this section is proving Proposition 2.2 , which shows that if two (possible non-complete) symplectic manifolds arise from different plurisubharmonic functions on the same complex manifold then their compactly supported symplectomorphism groups are weakly homotopy equivalent. This will be useful later as we will be able to identify the symplectomorphism groups of complements of ample divisors by identifying the complement up to biholomorphism.

Section 3 performs the computation of $\pi_{k}\left(\operatorname{Symp}_{c}\left(T^{*} \mathbb{R} \mathbb{P}^{2}\right)\right)$ and can actually be read independently of the rest of the paper. It closely follows [Sei98].

In Section 5 we carry out the computation in detail for $\mathbb{C}^{*} \times \mathbb{C}$. The only real difficulty is in proving weak contractibility of a space of symplectic 
spheres (playing the role of the divisor mentioned above). This proof is postponed to the end of the section and one crucial lemma is proved in the appendix.

We proceed to calculate the weak homotopy type of the symplectomorphism groups of $\mathbb{D}_{3}, \mathbb{D}_{4}$ and $\mathbb{D}_{5}$ in Section 6 and of the $A_{n}$-Milnor fibre in Section 7. Many of the proofs are similar to the case $\mathbb{C}^{*} \times \mathbb{C}$, so details are sketched noting where technicalities arise.

\section{Stein manifolds}

\subsection{Stein manifolds and Liouville flows.}

Definition 2.1 (Plurisubharmonic functions, Stein manifolds). A function $\phi: W \rightarrow[0, \infty)$ on a complex manifold $(W, J)$ is called plurisubharmonic if it is proper and if $\omega=-d(d \phi \circ J)$ is a positive $(1,1)$-form. A triple $(W, J, \phi)$ consisting of a complex manifold $(W, J)$ and a plurisubharmonic function $\phi$ on $(W, J)$ is called a Stein manifold. A sublevel set $\phi^{-1}[0, c]$ of a Stein manifold $(W, J, \phi)$ is called a Stein domain.

Given a Stein manifold $(W, J, \phi)$ one can associate to it:

- a one-form $\theta=-d \phi \circ J$ which is a primitive for the positive $(1,1)$-form $\omega$, and

- a vector field $Z$ which is $\omega$-dual to $\theta$.

We call $\theta$ the Liouville form and $Z$ the Liouville vector field. Let us abstract the notions of Liouville form and vector field further:

Definition 2.2. A Liouville manifold is a triple $(W, \theta, \phi)$ consisting of a smooth manifold $W$, a (Liouville) one-form $\theta$ on $W$ and a proper function $\phi: W \rightarrow[0, \infty)$ such that

- $\omega=d \theta$ is a symplectic form,

- there is a monotonic sequence $c_{i} \rightarrow \infty$ such that on the level sets $\phi^{-1}\left(c_{i}\right)$ the Liouville vector field $Z$ which is $\omega$-dual to $\theta$, satisfies $d \phi(Z)>0$ everywhere on $\phi^{-1}\left(c_{i}\right)$.

$(W, \theta, \phi)$ is said to have finite-type if there is a $k>0$ such that $d \phi(Z)>0$ at $x$ for any $x \in \phi^{-1}(k, \infty)$. It is said to be complete if the Liouville vector field is complete. A closed sublevel set $\phi^{-1}[0, k]$ of a Liouville manifold is called a Liouville domain.

Clearly, a Stein manifold inherits the structure of a Liouville manifold and a Stein domain inherits the structure of a Liouville domain.

On a Liouville domain, the negative-time Liouville flow always exists and one can use it to define an embedding Col: $(-\infty, 0] \times \partial W \rightarrow W$ with $\mathrm{Col}^{*} \theta=$ $\left.e^{r} \theta\right|_{\partial W}$ and $\mathrm{Col}_{*} \partial_{r}=Z$. We can therefore form the symplectic completion of $(W, \theta, \phi)$, which is a Liouville manifold: 
Definition 2.3 (Symplectic completion). Let $(W, \theta, \phi)$ be a Liouville domain with boundary $M$ and define $\alpha=\left.\theta\right|_{M}$. The symplectic completion $(\hat{W}, \hat{\theta})$ of $(W, \theta)$ is the manifold $\hat{W}=W \cup_{\text {Col }}(-\infty, \infty) \times M$ equipped with the one-form $\left.\hat{\theta}\right|_{W}=\theta,\left.\hat{\theta}\right|_{(-\infty, \infty) \times M}=e^{r} \alpha$. There is an associated symplectic form $\hat{\omega}=d \hat{\theta}$ and Liouville field $\left.\hat{Z}\right|_{W}=Z,\left.\hat{Z}\right|_{(-\infty, \infty) \times W}=\mathrm{Col}_{*} \partial_{r}$ whose flow exists for all times. We may take $\hat{\phi}$ to be any smooth extension of $\phi$ which agrees with $r$ outside some compact subset.

There is also a standard construction given a (possibly incomplete) finitetype Stein manifold $(W, J, \phi)$ to obtain a complete finite-type Stein manifold. Let $h$ denote the function $h(x)=e^{x}-1$.

Lemma 2.1 ([BC01, Lemma 3.1] and [SS05, Lemma 6]). Define $\phi_{h}:=$ $h \circ \phi .\left(W, J, \phi_{h}\right)$ is a complete Stein manifold of finite-type.

In fact, $\phi$ and $\phi_{h}$ have the same critical points. If $\theta$ and $Z$ are the Liouville form and vector field on $(W, J, \phi)$ then we write $\theta_{h}$ and $Z_{h}$ for the corresponding data on $\left(W, J, \phi_{h}\right)$. This construction is related to the symplectic completion of a sublevel Stein domain of $\phi$ :

Lemma 2.2. Let $k$ be such that $\phi^{-1}[0, k)$ contains all the critical points of $\phi$ and set $W_{k}=\phi^{-1}[0, k]$, which inherits the structure of a Liouville domain. Then $\left(\hat{W}_{k}, d \hat{\theta}\right)$ is symplectomorphic to $\left(W, d \theta_{h}\right)$.

Proof. Pick some $k^{\prime}>k$. Let $h_{0}$ be a function such that:

- $h_{0}(x)=h(x)$ if $x>k^{\prime}$,

- $h_{0}(x)=x$ if $x \leq k$ and

- $h_{0}^{\prime}(x)>0, h_{0}^{\prime \prime}(x)>0$ for all $x \in[0, \infty)$.

Define $h_{t}=t h+(1-t) h_{0}$. Now consider the functions $\phi_{t}=h_{t} \circ \phi$ on $W$ for $t \in[0,1]$. By Lemma 6 of [SS05] they are all plurisubharmonic functions making $\left(W, J, \phi_{t}\right)$ into a complete Stein manifold and they all have the same set of critical points. Let $\theta_{t}$ denote $d^{\mathrm{c}} \phi_{t}$. By Lemma 5 of $[\mathbf{S S 0 5}]$ there is a smooth family of diffeomorphisms $f_{t}: W \rightarrow W$ such that $f_{t}^{*} \theta_{t}=\theta_{0}+d R_{t}$ for some compactly supported function $R: W \times[0,1] \rightarrow \mathbb{R}$ with $R_{t}(x)=R(x, t)$.

By construction, $\phi_{1}=\phi_{h}$ and $\left.\phi_{0}\right|_{W_{k}}=\left.\phi\right|_{W_{k}}$. Therefore $\iota=\left.f_{1}\right|_{W_{k}}$ is a symplectic embedding of $W_{k}$ into $\left(W, d \theta_{h}\right)$ such that $\iota^{*} \theta_{h}=\left.\theta\right|_{W_{k}}+d R_{1}$ and there are no critical points of $\phi_{h}$ in the complement $W \backslash \iota\left(W_{k}\right)$.

We want to extend $\iota$ to a symplectomorphism $\tilde{\iota}:\left(\hat{W}_{k}, d \hat{\theta} \rightarrow\left(W, d \theta_{h}\right)\right.$. Begin by replacing $\hat{\theta}$ by $\hat{\theta}_{R}=\hat{\theta}+d R_{1}$. Of course $d \hat{\theta}=d \hat{\theta}_{R}$ still as $d R_{1}$ is closed. Let $Z_{R}$ be the $d \hat{\theta}$-dual Liouville field to $\hat{\theta}_{R}$. Then, on $\iota\left(W_{k}\right) \iota_{*} Z_{R}=$ $Z_{h}$. If $\Phi_{R}^{t}$ and $\Phi_{h}^{t}$ denote the time $t$ Liouville flows on $\left(\hat{W}_{k}, \hat{\theta}_{R}\right)$ and $\left(W, \theta_{h}\right)$, respectively, then

$$
\tilde{\iota}=\Phi_{h}^{t} \circ \iota \circ \Phi_{R}^{-t}: \hat{W}_{k} \rightarrow W
$$


defines the required symplectomorphism. Since we have used $\Phi_{R}^{t}$ and $\Phi_{R}^{-t}(x)=\Phi_{h}^{-t}(x)$ for $x \in W_{k}$ (by equality of Liouville vector fields), $\left.\tilde{\imath}\right|_{W_{k}}=\iota$.

We note another lemma for convenience.

Lemma 2.3. If $\phi_{1}$ and $\phi_{2}$ are complete plurisubharmonic functions on $(W, J)$ with finitely many critical points then $\left(W,-d d^{\mathrm{c}} \phi_{1}\right)$ is symplectomorphic to $\left(W,-d d^{\mathrm{c}} \phi_{2}\right)$.

Proof. This follows from applying Lemma 5 from [SS05] to the family $t \phi_{1}+$ $(1-t) \phi_{2}$ : the associated two-forms are all compatible with $J$ and hence all symplectic.

\subsection{Symplectomorphism groups.}

Definition 2.4. Let $(W, \omega)$ be a non-compact symplectic manifold and let $\mathcal{K}$ be the set of compact subsets of $W$. For each $K \in \mathcal{K}$ let $\operatorname{Symp}_{K}(W)$ denote the group of symplectomorphisms of $W$ supported in $K$, with the topology of $\mathcal{C}^{\infty}$-convergence. The group $\operatorname{Symp}_{c}(W, \omega)$ of compactly supported symplectomorphisms of $(W, \omega)$ is topologized as the direct limit of these groups under inclusions.

One important fact we will tacitly and repeatedly use in the sequel is:

Lemma 2.4. Let $C$ be compact and $f: C \rightarrow \operatorname{Symp}_{\mathrm{c}}(W, \omega)$ be continuous. Then the image of $f$ is contained in $\operatorname{Symp}_{K}(W, \omega)$ for some $K$.

Let us restrict attention to the class of Liouville manifolds $(W, \theta, \phi)$ which are of finite-type and satisfy:

Condition 1. There is a $T \in[0, \infty], a K \in[0, \infty)$ and an exhausting function $f:[0, T) \rightarrow[K, \infty)$ such that:

- the Liouville flow of any point on $\phi^{-1}(K)$ is defined until time $T$,

- for all $t \in[0, T)$, the Liouville flow defines a diffeomorphism $\phi^{-1}(K) \rightarrow \phi^{-1}(f(t))$.

Examples of such Liouville manifolds include Stein manifolds (where the Liouville flow is the gradient flow of $\phi$ with respect to the Kähler metric) and symplectic completions of Liouville domains.

A Liouville manifold $(W, \theta, \phi)$ comes with a canonical family of compact subsets $W_{r}=\phi^{-1}[0, r]$ with natural inclusion maps $\iota_{r, R}: W_{r} \hookrightarrow W_{R}$ for all $r<R$. This family is exhausting, that is $\bigcup_{r \in[0, \infty)} W_{r}=W$. When $(W, \theta, \phi)$ satisfies Condition 1 we can use this family to better understand the homotopy type of the symplectomorphism group $\operatorname{Symp}_{\mathrm{c}}(W, d \theta)$.

Proposition 2.1. If $(W, \theta, \phi)$ is a Liouville manifold satisfying Condition 1 and $K \in[0, \infty)$ is such that $W_{K}=\phi^{-1}[0, K)$ contains all critical points of $\phi$ then $\operatorname{Symp}_{\mathrm{c}}\left(W_{K},\left.d \theta\right|_{W_{K}}\right)$ is weakly homotopy equivalent to $\operatorname{Symp}_{\mathrm{c}}(W, d \theta)$. 
Proof. Let $\Phi^{t}$ denote the time $t$ Liouville flow of $Z$ where that is defined. Let $L_{R-r}: \operatorname{Symp}_{W_{R}}(W, d \theta) \rightarrow \operatorname{Symp}_{W_{r}}(W, d \theta)$ be the map:

$$
L_{R-r}(\psi)=\Phi^{r-R} \circ \psi \circ \Phi^{R-r} .
$$

The inclusions $\iota_{r, R}$ induce inclusions

$$
\iota_{r, R}^{S}: \operatorname{Symp}_{W_{r}}(W, d \theta) \hookrightarrow \operatorname{Symp}_{W_{R}}(W, d \theta)
$$

for $r, R \geq K$. The maps $L_{R-r}$ are homotopy inverses for $\iota_{r, R}^{S}$. Similarly, the inclusion

$$
\iota_{\mathrm{c}}^{S}: \operatorname{Symp}_{\mathrm{c}}\left(W_{K},\left.d \theta\right|_{W_{K}}\right) \rightarrow \operatorname{Symp}_{W_{K}}(W, d \theta)
$$

has $L_{\epsilon}$ (for any small $\epsilon$ ) as a homotopy inverse.

We wish to prove that the inclusion

$$
\iota: \operatorname{Symp}_{\mathrm{c}}\left(W_{K},\left.d \theta\right|_{W_{K}}\right) \rightarrow \operatorname{Symp}_{\mathrm{c}}(W, d \theta)
$$

is a weak homotopy equivalence. To see that the maps

$$
\iota_{\star}: \pi_{n}\left(\operatorname{Symp}_{\mathrm{c}}\left(W_{K},\left.d \theta\right|_{W_{K}}\right)\right) \rightarrow \pi_{n}\left(\operatorname{Symp}_{\mathrm{c}}(W, d \theta)\right)
$$

are surjective, it suffices to note that the image of any $f: S^{n} \rightarrow \operatorname{Symp}_{\mathrm{c}}(W, d \theta)$ lands in $\operatorname{Symp}_{W_{R}}(W, d \theta)$ for some $R$ and that $\iota_{0, R} \circ \iota_{\mathrm{c}}$ is a homotopy equivalence. To see injectivity, any homotopy $h$ between maps $f_{1}, f_{2}$ : $S^{n} \rightarrow \operatorname{Symp}_{\mathrm{c}}\left(W_{K},\left.d \theta\right|_{W_{K}}\right)$ through maps $S^{n} \rightarrow \operatorname{Symp}_{\mathrm{c}}(W, d \theta)$ lands in some $\operatorname{Symp}_{W_{R}}(W, d \theta)$ and $\iota_{0, R} \circ \iota_{\mathrm{c}}$ is a homotopy equivalence so $h$ is homotopic to a homotopy $f_{1} \simeq f_{2}$ in $\operatorname{Symp}_{\mathrm{c}}\left(W_{K},\left.d \theta\right|_{W_{K}}\right)$.

Proposition 2.2. If $(W, J)$ is a complex manifold with two finite-type Stein structures $\phi_{1}$ and $\phi_{2}$ then $\operatorname{Symp}_{\mathrm{c}}\left(W,-d d^{\mathrm{c}} \phi_{1}\right)$ and $\operatorname{Symp}_{\mathrm{c}}\left(W,-d d^{\mathrm{c}} \phi_{2}\right)$ are weakly homotopy equivalent.

Proof. Let $\omega_{i}=-d d^{\mathrm{c}} \phi_{i}$. By Proposition $2.1, \operatorname{Symp}_{\mathrm{c}}\left(W, \omega_{i}\right)$ is weakly homotopy equivalent to $\operatorname{Symp}_{\mathrm{c}}\left(W_{K_{i}},\left.\omega_{i}\right|_{W_{K_{i}}}\right)$ for some $K_{i}$. Again by Proposition 2.1 , this is weakly homotopy equivalent to $\operatorname{Symp}_{\mathrm{c}}\left(\hat{W}_{K_{i}}, \hat{\omega}_{i}\right)$. Now by Lemma 2.2, this is isomorphic to $\operatorname{Symp}_{\mathrm{c}}\left(W,-d d^{\mathrm{c}}\left(\phi_{i}\right)_{h}\right)$. But by Lemma 2.3, $\left(W,-d d^{\mathrm{c}}\left(\phi_{1}\right)_{h}\right)$ is symplectomorphic to $\left(W,-d d^{\mathrm{c}}\left(\phi_{2}\right)_{h}\right)$ (since $\left(\phi_{i}\right)_{h}$ is complete). This proves the proposition.

\section{3. $\operatorname{Symp}_{\mathrm{c}}\left(T^{*} \mathbb{R} \mathbb{P}^{2}\right)$}

Theorem 1.5. Let $\omega$ be the canonical symplectic form on the cotangent bundle of $\mathbb{R P}^{2}$. The group of compactly supported symplectomorphisms $\operatorname{Symp}_{\mathrm{c}}\left(T^{*} \mathbb{R P}^{2}\right)$ is weakly homotopy equivalent to $\mathbb{Z}$.

Proof. The proof is an adaptation of Seidel's proof [Sei98] for $T^{*} S^{2}$. The two-to-one cover $S^{2} \rightarrow \mathbb{R P}^{2}$ differentiates to a two-to-one cover of tangent bundles. Identifying tangent and cotangent bundles via the round metric, we 
get a two-to-one cover $T^{*} S^{2} \rightarrow T^{*} \mathbb{R P}^{2}$. Restrict this to a two-to-one cover of the unit disc subbundles $U^{*}\left(S^{2}\right) \subset T^{*} S^{2}$ and $U^{*}\left(\mathbb{R} \mathbb{P}^{2}\right) \subset T^{*} \mathbb{R} \mathbb{P}^{2}$ :

$$
\pi: U^{*}\left(S^{2}\right) \rightarrow U^{*}\left(\mathbb{R P}^{2}\right) .
$$

$\pi$ intertwines the cogeodesic flows on these cotangent disc bundles. Since the cogeodesic flows are periodic, one can symplectically cut along them and $\pi$ extends to a branched cover of the compactifications:

$$
S^{2} \times S^{2} \rightarrow \mathbb{C P}^{2}
$$

branched along the diagonal $\Delta \subset S^{2} \times S^{2}$ (which maps one-to-one onto a conic $C$ in $\mathbb{C P}^{2}$ ). Here $\Delta$ and $C$ are the reduced loci of the symplectic cut.

Let $\iota: S^{2} \times S^{2} \rightarrow S^{2} \times S^{2}$ be the involution which swaps the two $S^{2}$ factors. This is the deck transformation of the branched cover. Let:

- $\mathcal{S}_{0}^{\iota}$ denote the group of $\iota$-equivariant symplectomorphisms of $S^{2} \times S^{2}$ which fix $\Delta$ pointwise and act trivially on the homology of $S^{2} \times S^{2}$;

- $\mathcal{S}_{1}^{\iota} \subset \mathcal{S}_{0}^{\iota}$ denote the subgroup of symplectomorphisms compactly supported in the complement of $\Delta$; and

- $\mathcal{S}$ denote the group of symplectomorphisms of $\mathbb{C P}^{2}$ compactly supported in the complement of $C$. This is homotopy equivalent to the group we are interested in.

An element $\tilde{\phi} \in \mathcal{S}_{1}^{\iota}$ descends to an element $\phi \in \mathcal{S}$. $\tilde{\phi}$ is the only $\iota$-equivariant symplectomorphism of $S^{2} \times S^{2}$ which acts trivially on homology and descends to $\phi$, so the correspondence $\tilde{\phi} \rightarrow \phi$ is a homeomorphism $\mathcal{S}_{1}^{\iota} \rightarrow \mathcal{S}$. We have therefore reduced the proposition to computing the weak homotopy type of $\mathcal{S}_{1}^{\iota}$.

Let $\mathcal{G}$ be the group of gauge transformations of the symplectic normal bundle to $\Delta \subset S^{2} \times S^{2}$. By a standard argument [Sei08] this is homotopy equivalent to $S^{1}$. Let $\mathcal{S}_{0}^{\iota} \rightarrow \mathcal{G}$ be the map taking an $\iota$-equivariant symplectomorphism fixing $\Delta$ to its derivative along the normal bundle of $\Delta$. This is a fibration whose fibre is weakly homotopy equivalent to $\mathcal{S}_{1}^{\iota}$. The proposition will follow from the long exact sequence of this fibration if we can show that $\mathcal{S}_{0}^{\iota}$ is contractible. This is where Gromov's theorem comes in.

Let $J_{0}$ denote the product complex structure on $S^{2} \times S^{2}$. Let $\mathcal{J}_{0}^{\iota}$ denote the space of $\omega$-compatible almost complex structures $J$ on $S^{2} \times S^{2}$ such that:

- $J \circ \iota_{*}=\iota_{*} \circ J$ and

- $J$ restricted to $T \Delta$ is equal to $J_{0}$.

Lemma 3.1. $\mathcal{J}_{0}^{\iota}$ is contractible.

Proof. Recall that if $g$ is a metric on a manifold $X$ and $\omega$ a symplectic form then the endomorphism $A \in \operatorname{End}(T X)$ defined by

$$
\omega(\cdot, A \cdot)=g(\cdot, \cdot)
$$


satisfies $A^{\dagger}=-A$, so $A^{\dagger} A$ is symmetric and positive definite. Let $P A^{\dagger} P^{-1}=$ $\operatorname{diag}\left(\lambda_{1}, \ldots, \lambda_{n}\right)$ be the diagonalization of $A^{\dagger} A$. Define the square $\operatorname{root} \sqrt{A^{\dagger} A}$ to be the matrix $P^{-1} \operatorname{diag}\left(\sqrt{\lambda_{1}}, \ldots, \sqrt{\lambda_{n}}\right) P$. Then $J_{A}={\sqrt{A^{\dagger} A}}^{-1} A$ is an almost complex structure on $X$ compatible with $\omega$.

Let $J$ be an almost complex structure on $S^{2} \times S^{2}$ compatible with the product form $\omega$. This defines a metric $g_{0}$ via $g_{0}(\cdot, \cdot)=\omega(\cdot, J \cdot)$. Similarly, $\iota^{*} J$ is an $\omega$-compatible almost complex structure which defines a metric $g_{1}$. The space of metrics is convex and the metric $g_{t}=(1-t) g_{0}+t g_{1}$ defines a family of endomorphisms $A_{t}$ as above interpolating between $J$ and $\iota^{*} J$. Now $J_{A_{t}}$ is a family of almost complex structures interpolating between $J$ and $\iota^{*} J$ and $J_{A_{1 / 2}}$ is $\iota_{*}$-invariant. Hence the contractible space of all $\omega$-compatible almost complex structures (equal to $J_{0}$ along $\Delta$ ) deformation retracts onto the space of $\iota_{*}$-equivariant ones.

We now prove that $\mathcal{S}_{0}^{\iota}$ is contractible. There is a map $A: \mathcal{S}_{0}^{\iota} \rightarrow \mathcal{J}_{0}^{\iota}$ which sends $\phi$ to $\phi_{*} J_{0}$. The following argument constructs a left inverse $B$ for $A$.

Gromov's theorem gives $J$-holomorphic foliations $\mathcal{F}_{1}^{J}$ and $\mathcal{F}_{2}^{J}$ for each $J \in \mathcal{J}_{0}^{\iota}$. In fact, the two foliations must be conjugate under $\iota$ since $\iota \mathcal{F}_{1}^{J}$ is certainly a foliation by $J$-holomorphic spheres in the homology class $\mathcal{F}_{2}^{J}$ and positivity of intersections implies it is the unique one. We also know that, for any $J \in \mathcal{J}_{0}^{\iota}, \Delta$ is $J$-holomorphic so each leaf of $\mathcal{F}_{i}^{J}$ intersects $\Delta$ in a single point. Define a (manifestly $\iota$-equivariant) self-diffeomorphism $\psi_{J}$ of $S^{2} \times S^{2}$ by sending a point $p$ to the pair $\left(\Lambda_{1}(p) \cap \Delta, \Lambda_{2}(p) \cap \Delta\right)$ where $\Lambda_{i}(p)$ denotes the unique leaf of $\mathcal{F}_{i}^{J}$ passing through $p . \psi_{J}$ conjugates the pair $\left(\mathcal{F}_{1}^{J}, \mathcal{F}_{2}^{J}\right)$ with the standard pair $\left(\mathcal{F}_{1}^{J_{0}}, \mathcal{F}_{2}^{J_{0}}\right)$. Since these standard foliations are $\omega$-orthogonal, it is not hard to see that $\psi_{J}^{*} \omega$ is $J$-tame. Define $\omega_{t}=t \psi_{J}^{*} \omega+(1-t) \omega$, so that

$$
\dot{\omega}_{t}=\psi_{J}^{*} \omega-\omega
$$

Since $\psi_{J}$ acts trivially on homology this expression is exact and there exists $\sigma$ such that $d \sigma=\psi_{J}^{*} \omega-\omega$. If $X_{t}$ is $\omega_{t}$-dual to $\sigma$ then the time $t$ flow of $X_{t}$ defines a sequence of diffeomorphisms $\psi_{t}$ such that $\psi_{0}=$ id and $\psi_{t}^{*} \omega_{t}=\omega$. Therefore $\psi_{J, t}=\psi_{J} \circ \psi_{t}$ is an isotopy from $\psi_{J}$ to a symplectomorphism $\psi_{J, 1}$ of $\omega$. We want $\psi_{J, t}$ to have the following properties:

- $\psi_{J, t}$ is $\iota$-equivariant for all $t$,

- $\psi_{J, t}$ fixes $\Delta$ for all $t$ and

- if $J=\phi_{*} J_{0}$ then $\psi_{J, 1}=\phi$.

These can be achieved by suitable choice of $\sigma$. The first two follow if we require $\sigma$ to be $\iota^{*}$-invariant and to vanish when restricted to $\left.T X\right|_{\Delta}$. These properties will be achieved momentarily (in a manner such that $\sigma$ varies smoothly with $J$ ). The third property follows if we can take $\sigma \equiv 0$ whenever $d \sigma \equiv 0$; for then if $J=\phi_{*} J_{0}$, the foliations $\mathcal{F}_{i}^{J}$ are just $\phi\left(\mathcal{F}_{i}^{J_{0}}\right)$ so $\psi_{J}=\phi$ and $\dot{\omega}_{t}=0=d \sigma$. Taking $\sigma=0$ yields $\psi_{J, 1}=\psi_{J, 0}=\phi$. 
Fix a metric $g$ and let $\sigma^{\prime}$ be the unique $g$-coexact one-form for which $d \sigma^{\prime}=\dot{\omega}_{t}$ (which exists and varies smoothly with $J$ by Hodge theory). In order to make sure $\psi_{J, t}$ fixes $\Delta$, we want $\sigma$ to vanish on the bundle $\left.T X\right|_{\Delta}$. Since $\left.\psi_{J}\right|_{\Delta}=\mathrm{id}, d \sigma^{\prime}=0$ along $\Delta$. In particular, we may choose a function $f$ on $\Delta$ with $d f=\left.\sigma^{\prime}\right|_{\Delta}\left(\right.$ as $\left.H^{1}(\Delta ; \mathbb{R})=0\right)$ and this choice is unique if we require $\int_{\Delta} f \omega=0$. Fix an $\epsilon$ such that the map $\exp _{g}: U_{\epsilon} \rightarrow X$ is injective on the radius- $\epsilon$ subbundle $U_{\epsilon}$ of the normal bundle $T \Delta^{\perp}$. Define

$$
\tilde{f}(\exp (q, v))=f(\exp (q, 0))+\sigma_{\exp (q, 0)}^{\prime}\left(\exp _{*} v\right) .
$$

This satisfies $d \tilde{f}=\sigma^{\prime}$ when restricted to $\left.T X\right|_{\Delta}$. Fix a cut-off function $\eta$ which equals 1 inside the radius- $\epsilon / 2$ subbundle $U_{\epsilon / 2}$ and equals zero near the boundary of $U_{\epsilon}$. The function $F=\eta \tilde{f}$ is now globally defined and still satisfies $d F=\sigma^{\prime}$ when restricted to $\left.T X\right|_{\Delta}$. The one-form

$$
\sigma:=\frac{\left(\sigma^{\prime}-d F\right)+\iota^{*}\left(\sigma^{\prime}-d F\right)}{2}
$$

is now $\iota^{*}$-invariant, vanishes along $\Delta$ and satisfies $d \sigma=\dot{\omega}_{t}$.

We finally note that $\sigma$ varies smoothly with $J$ and that the unique coexact $\sigma$ with $d \sigma=0$ is $\sigma=0$, so the third property holds (by Hodge theory).

This proves that $\mathcal{S}_{0}^{\iota}$ is homotopy equivalent to $\mathcal{J}_{0}^{\iota}$ and hence contractible by Lemma 3.1. The proposition now follows from the homotopy long exact sequence of the fibration $\mathcal{S}_{0}^{\iota} \rightarrow \mathcal{G}$, whose fibre is weakly equivalent to $\mathcal{S}_{1}^{\iota}$ by the symplectic neighbourhood theorem.

Remark 3.1. Since all geodesics of $\mathbb{R P}^{2}$ are closed one can define a Dehn twist in a Lagrangian $\mathbb{R P}^{2}$ just as one does for $S^{2}$. This is actually the generator of the symplectic mapping class group $\mathbb{Z}$, see [Sei98].

\section{Groups associated to configurations of spheres}

This section reviews the topology of some well-known (Fréchet-) Lie groups which will crop up frequently later.

4.1. Gauge transformations. Let $C \subset X$ be an embedded symplectic two-sphere in a symplectic four-manifold and fix a set $\left\{x_{1}, \ldots, x_{k}\right\}$ of distinct points of $C$. The normal bundle $\nu=T C^{\omega \perp}$ is an $\operatorname{SL}(2, \mathbb{R})$-bundle over $C$. We will be interested in the group $\mathcal{G}_{k}$ of symplectic gauge transformations of $\nu$ which equal the identity at $x_{1}, \ldots, x_{k}$. This will arise when we consider symplectomorphisms of $X$ which fix $C$ pointwise and are required to equal the identity at the $k$ specified points on $C$.

We first observe that $\mathrm{SL}(2, \mathbb{R})$ deformation retracts to the subgroup $U(1)$ which is homeomorphic to a circle. The map

$$
\mathrm{ev}_{x_{1}}: \mathcal{G}_{0} \rightarrow \mathrm{SL}(2, \mathbb{R})
$$


taking a gauge transformation to its value at the point $x_{1}$ is a fibration whose fibre is $\mathcal{G}_{1}$. By definition, $\mathcal{G}_{1}$ is the space of based maps $S^{2} \rightarrow \operatorname{SL}(2, \mathbb{R})$ so

$$
\pi_{i}\left(\mathcal{G}_{1}\right)=\pi_{0}\left(\operatorname{Map}\left(S^{2+i}, S L(2, \mathbb{R})\right)\right)=\pi_{0}\left(\operatorname{Map}\left(S^{2+i}, S^{1}\right)\right)=H^{1}\left(S^{2+i} ; Z Z\right)=0
$$

and $\mathcal{G}_{1}$ is weakly contractible. By the long exact sequence of the fibration $\mathrm{ev}_{x_{1}}$ we see that $\mathrm{ev}_{x_{1}}$ is a weak homotopy equivalence.

Take the map $\mathrm{ev}_{x_{1}} \times \cdot \times \mathrm{ev}_{x_{i}}: \mathcal{G}_{1} \rightarrow \mathrm{SL}(2, \mathbb{R})^{i}$. This is again a fibration whose fibre is $\mathcal{G}_{i}$ and whose total space is contractible. The homotopy long exact sequence of this fibration implies that $\pi_{0}\left(\mathcal{G}_{i}\right)=\mathbb{Z}^{i-1}$ for $i \geq 1$ and $\pi_{j}\left(\mathcal{G}_{i}\right)=0$ for $j>0$.

We can identify generators for $\pi_{0}\left(\mathcal{G}_{k}\right)$ as follows. For each point $x_{i}$ the map $\mathrm{ev}_{x_{i}}: \mathcal{G}_{k-1} \rightarrow \mathrm{SL}(2, \mathbb{R})$ yields a connecting homomorphism $\mathbb{Z}=$ $\pi_{1}(\mathrm{SL}(2, \mathbb{R})) \rightarrow \pi_{0}\left(\mathcal{G}_{k}\right)$. The symplectic form gives an orientation of $\mathrm{SL}(2, \mathbb{R})$ so there is a preferred element $1 \in \mathbb{Z}$. Let $g_{C}\left(x_{i}\right) \in \mathcal{G}_{k}$ be the image of this element in $\pi_{0}\left(\mathcal{G}_{k}\right)$. They are not independent, but they are canonical and they generate.

To summarize:

$$
\begin{aligned}
& \mathcal{G}_{0} \simeq S^{1} \\
& \mathcal{G}_{1} \simeq \star \\
& \mathcal{G}_{k} \simeq \mathbb{Z}^{k-1}, \quad k>1 .
\end{aligned}
$$

4.2. Surface symplectomorphisms. Once again, let $C \subset X$ be an embedded symplectic two-sphere and $\left\{x_{1}, \ldots, x_{k}\right\}$ a set of $k$ distinct points. Let $\operatorname{Symp}\left(C,\left\{x_{i}\right\}\right)$ denote the group of symplectomorphisms of $C$ fixing the points $x_{i}$. Since $\operatorname{Symp}(C)$ acts $N$-transitively on points for any $N$, we may as well write $\operatorname{Symp}(C, k)$. Moser's theorem tells us that $\operatorname{Symp}(C, k)$ is a deformation retract of the group $\mathcal{D}_{k}$ of diffeomorphisms of $S^{2}$ fixing $k$ points. Using techniques of Earle and Eells [EE67] coming from Teichmüller theory, one can prove:

- $\mathcal{D}_{1} \simeq S^{1}$,

- $\mathcal{D}_{2} \simeq S^{1}$,

- $\mathcal{D}_{3} \simeq \star$

- $\mathcal{D}_{5} \simeq P \operatorname{Br}\left(S^{2}, 5\right) / \mathbb{Z} / 2$, where $P \operatorname{Br}\left(S^{2}, 5\right)$ is the pure braid group for five-strands on $S^{2}$ and the $\mathbb{Z} / 2$ is generated by a full twist (see Section $6.1)$.

Here the $S^{1}$ in $\mathcal{D}_{1}$ can be thought of as rotating around the fixed point. $\mathcal{D}_{2}$ has a map to $S^{1} \times S^{1}$ measuring the angle of rotation of a diffeomorphism about the two fixed points $p_{1}$ and $p_{2}$. The kernel of this map is the group $\mathcal{D}_{2}^{\mathrm{c}}$ of compactly supported diffeomorphisms of $S^{2} \backslash\left\{p_{1}, p_{2}\right\}$, which is weakly equivalent to $\mathbb{Z}$ generated by a Dehn twist in a simple closed curve generating the fundamental group of $S^{2} \backslash\left\{p_{1}, p_{2}\right\}$. In the homotopy long exact sequence 
of the fibration $\mathcal{D}_{2}^{c} \rightarrow \mathcal{D}_{2} \rightarrow\left(S^{1}\right)^{2}$, one obtains:

$$
1 \rightarrow \pi_{1}\left(\mathcal{D}_{2}\right) \rightarrow \mathbb{Z}^{2} \rightarrow \mathbb{Z} \rightarrow \pi_{0}\left(\mathcal{D}_{2}\right) \rightarrow 1 .
$$

The Dehn twist is the image of $(1,0)$ and $(0,1)$ under the map $\mathbb{Z}^{2} \rightarrow \mathbb{Z}$, so $\mathcal{D}_{2} \simeq S^{1}$. If $p_{1}$ and $p_{2}$ are antipodal, a $2 \pi$ rotation around the axis through them gives a non-trivial loop.

4.3. Configurations of spheres. We will frequently meet the situation where there is a configuration of embedded symplectic two-spheres $C=$ $C_{1} \cup \cdots \cup C_{n} \subset X$ in a four-manifold. Write $I$ for the set of intersection points among the components. Suppose that there are no triple intersections among components and that all intersections are transverse. Let

- $\operatorname{Stab}(C)$ denote the group of symplectomorphisms of $X$ fixing the components of $C$ pointwise;

- $k_{i}$ denote the number of intersection points in $I \cap C_{i}$ and $\operatorname{Symp}\left(C_{i}, k_{i}\right)$ denote the group of symplectomorphisms of components of $C$ fixing all the intersection points. Write $\operatorname{Symp}(C)$ for the product $\prod_{i=1}^{n} \operatorname{Symp}\left(C_{i}, k_{i}\right)$; and

- $\mathcal{G}\left(C_{i}\right)$ denote the group of gauge transformations of the normal bundle to $C_{i} \subset X$ which equal the identity at the $k_{i}$ intersection points (so $\left.\mathcal{G}\left(C_{i}\right) \cong \mathcal{G}_{k_{i}}\right)$. Write $\mathcal{G}$ for the product $\prod_{i=1}^{n} \mathcal{G}\left(C_{i}\right)$.

Let us write $\operatorname{Stab}^{0}(C)$ for the kernel of the map

$$
\begin{aligned}
\operatorname{Stab}(C) & \rightarrow \operatorname{Symp}(C), \\
\phi & \mapsto\left(\left.\phi\right|_{C_{1}}, \ldots,\left.\phi\right|_{C_{n}}\right) .
\end{aligned}
$$

We will need to understand the homotopy long exact sequence of the fibration

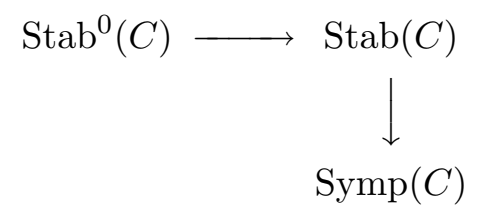

namely

$$
\cdots \rightarrow \pi_{1}(\operatorname{Symp}(C)) \rightarrow \pi_{0}\left(\operatorname{Stab}^{0}(C)\right) \rightarrow \pi_{0}(\operatorname{Stab}(C)) \rightarrow \cdots .
$$

There is a map $\operatorname{Stab}^{0}(C) \rightarrow \mathcal{G}$ which sends a symplectomorphism $\phi$ to the induced map on the normal bundles of components of $C$. We can understand the composite

$$
\pi_{1}(\operatorname{Symp}(C)) \rightarrow \pi_{0}\left(\operatorname{Stab}^{0}(C)\right) \rightarrow \pi_{0}(\mathcal{G})
$$

by thinking purely locally in a neighbourhood of $C$.

To see this, note that $\pi_{1}(\operatorname{Symp}(C))$ is only non-trivial if $C$ contains components with $n_{i}=1$ or 2 marked points. Suppose $n_{i}=1$. There is a Hamiltonian circle action which rotates $C_{i}$ around the marked point, giving 
a generating loop $\gamma$ in $\pi_{1}\left(\operatorname{Symp}\left(C_{i}, n_{i}\right)\right)$. Pull this back to a Hamiltonian circle action on the normal bundle of $C_{i}$ in $X$. By the symplectic neighbourhood theorem this is a local model for $X$ near $C_{i}$, so by cutting off the Hamiltonian at some radius in the normal bundle one obtains a path $\phi_{t}$ in $\operatorname{Symp}(X)$ consisting of symplectomorphisms which are supported in a neighbourhood of $C_{i}$ (see figure). The symplectomorphism $\phi_{t}$ can be assumed to fix $C$ as a set (indeed it just rotates the component $C_{i}$ ) and $\phi_{2 \pi}$ fixes $C$ pointwise, i.e., $\phi_{2 \pi} \in \operatorname{Stab}^{0}(C)$. By definition, $\phi_{2 \pi}$ represents the image of $\gamma \in \pi_{1}(\operatorname{Symp}(C))$ under the boundary map $\pi_{1}(\operatorname{Symp}(C)) \rightarrow \pi_{0}\left(\operatorname{Stab}^{0}(C)\right)$ of the long exact sequence above. A similar story holds when $n_{i}=2$. The following lemma is immediate from the definitions.

Lemma 4.1. The image of $\phi_{2 \pi}$ under the map $\pi_{0}\left(\operatorname{Stab}^{0}(C)\right) \rightarrow \pi_{0}(\mathcal{G})$ is

- $\left(n_{i}=1:\right) g_{C_{j}}(x) \in \pi_{0}\left(\mathcal{G}\left(C_{j}\right)\right)$ where $x \in C_{i} \cap C_{j}$,

- $\left(n_{i}=2:\right) g_{C_{j}}(x) g_{C_{k}}(y) \in \pi_{0}\left(\mathcal{G}\left(C_{j}\right)\right) \times \pi_{0}\left(\mathcal{G}\left(C_{k}\right)\right)$ where $x \in C_{i} \cap C_{j}$ and $y \in C_{i} \cap C_{k}$.

4.4. Banyaga's isotopy extension theorem. We will make constant recourse to the following ( $k$-parameter version of a) theorem of Banyaga:

Theorem 4.1 (Banyaga's isotopy extension theorem, see [MS05, p. 98]). Let $(X, \omega)$ be a compact symplectic manifold and $C_{s} \subset X$ be a $k$-parameter family of compact subsets $\left(s \in[0,1]^{k}\right)$. Let $\phi_{t, s}: U \rightarrow X$ be a k-parameter family of symplectic isotopies of open sets such that $U_{s}:=\phi_{0, s}(U)$ is a neighbourhood of $C_{s}$ and assume that for all $s$

$$
H^{2}\left(X, C_{s} ; \mathbb{R}\right)=0 .
$$

Then there are neighbourhoods $\mathcal{N}_{s} \subset U_{s}$ of $C_{s}$ and a $k$-parameter family of symplectic isotopies $\psi_{t, s}: X \rightarrow X$ such that $\left.\psi_{t, s}\right|_{\mathcal{N}_{s}}=\left.\phi_{t, s}\right|_{\mathcal{N}_{s}}$ for all $t$ and $s$.

The proof uses a standard Moser-type argument. This theorem will be used to prove the homotopy lifting property on a number of occasions.

\section{5. $\operatorname{Symp}_{\mathbf{c}}\left(\mathbb{C}^{*} \times \mathbb{C}\right)$}

In this section we prove the following theorem:

Theorem 1.6. Let $\omega$ be the product symplectic form on $\mathbb{C}^{*} \times \mathbb{C}$. The group of compactly supported symplectomorphisms $\operatorname{Symp}_{\mathrm{c}}\left(\mathbb{C}^{*} \times \mathbb{C}\right)$ is weakly contractible.

5.1. Proof of Theorem 1.6. We define three holomorphic spheres in $X=$ $\mathbb{C P}^{1} \times \mathbb{C P}^{1}$ :

$$
\begin{aligned}
& C_{1}=\{0\} \times \mathbb{C P}^{1}, \\
& C_{2}=\{\infty\} \times \mathbb{C P}^{1}, \\
& C_{3}=\mathbb{C P}^{1} \times\{\infty\} .
\end{aligned}
$$


Consider the complements

$$
U=X \backslash C_{1} \cup C_{2} \cup C_{3}, \quad \quad U^{\prime}=X \backslash C_{2} \cup C_{3} .
$$

These are biholomorphic to $\mathbb{C}^{*} \times \mathbb{C}$ and $\mathbb{C}^{2}$, respectively. The split symplectic form $\omega$ on $X$ restricts to symplectic forms $\left.\omega\right|_{U}$ and $\left.\omega\right|_{U^{\prime}}$ which are both Stein for the standard (product) complex structures. By Proposition 2.2,

$$
\operatorname{Symp}_{\mathrm{c}}(U) \simeq \operatorname{Symp}_{\mathrm{c}}\left(\mathbb{C}^{*} \times \mathbb{C}\right), \quad \operatorname{Symp}_{\mathrm{c}}\left(U^{\prime}\right) \simeq \operatorname{Symp}_{\mathrm{c}}\left(\mathbb{C}^{2}\right)
$$

and by a theorem of Gromov $[\mathbf{G r o 8 5}], \operatorname{Symp}_{\mathrm{c}}\left(\mathbb{C}^{2}\right) \simeq \star$.

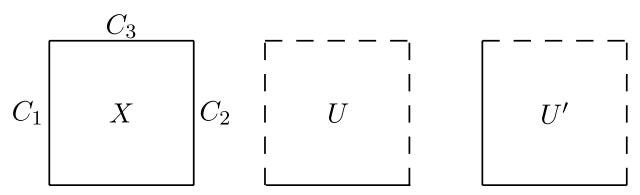

We will now define a space on which $\operatorname{Symp}_{\mathrm{c}}\left(U^{\prime}\right)$ acts. Let $\mathcal{J}$ denote the space of $\omega$-compatible almost complex structures on $X$.

Definition 5.1. A standard configuration in $X$ is an embedded symplectic sphere $S$ satisfying the following properties:

- $S$ is homologous to $C_{1}$,

- $S$ is disjoint from $C_{2}$,

- there exists a $J \in \mathcal{J}$ making $S, C_{2}$ and $C_{3}$ simultaneously J-holomorphic,

- there exists a neighbourhood $\nu$ of $C_{3}$ such that $\nu \cap S$ is equal to $\nu \cap C_{1}$.

Let $\mathcal{C}_{0}$ denote the space of standard configurations, topologized as a subset of the quotient

$$
\operatorname{Map}\left(\mathbb{C P}^{1}, X\right) / \operatorname{Diff}\left(\mathbb{C P}^{1}\right),
$$

where $\operatorname{Map}\left(\mathbb{C P}^{1}, X\right)$ and $\operatorname{Diff}\left(\mathbb{C P}^{1}\right)$ are given the $\mathcal{C}^{\infty}$-topology.

The first important fact about this space is the following proposition whose proof is postponed to Section 5.2:

Proposition 5.1. $\mathcal{C}_{0}$ is weakly contractible.

Given this proposition, we observe that

Lemma 5.1. $\operatorname{Symp}_{\mathrm{c}}\left(U^{\prime}\right)$ acts transitively on $\mathcal{C}_{0}$.

Proof. Since $\mathcal{C}_{0}$ is weakly contractible it is path-connected. If $S_{0}$ and $S_{1}$ are two standard configurations let $S_{t}$ be a path connecting them. Then

$$
T_{t}=S_{t} \cup C_{2} \cup C_{3}
$$

is an isotopy of configurations of symplectic spheres. Since $S_{t}$ is a standard configuration for each $t$ the isotopy $T_{t}$ extends to an isotopy of a neighbourhood of $T_{t}$ by the symplectic neighbourhood theorem. We may assume this isotopy fixes a neighbourhood of $C_{2} \cup C_{3}$ pointwise. Since $H^{2}\left(X, T_{t} ; \mathbb{R}\right)=0$, 
Banyaga's Theorem 4.1 above implies that this isotopy extends to a path $\psi_{t}$ in $\operatorname{Symp}(X)$. By construction $\psi_{t}$ is the identity on a neighbourhood of $C_{2} \cup C_{3}$. Hence $\psi_{1} \in \operatorname{Symp}_{\mathrm{c}}\left(U^{\prime}\right)$ sends $S_{0}$ to $S_{1}$, proving transitivity.

Moreover, the map $\operatorname{Symp}_{\mathrm{c}}\left(U^{\prime}\right) \rightarrow \mathcal{C}_{0}=\operatorname{Orb}\left(C_{1}\right)$ is a fibration by Banyaga's theorem. We identify the stabilizer:

Lemma 5.2. The stabilizer $\operatorname{Stab}\left(C_{1}\right)$ of $C_{1}$ under this action is weakly homotopy equivalent to $\operatorname{Symp}_{\mathrm{c}}(U)$.

Proof. Since a symplectomorphism $\phi \in \operatorname{Symp}_{\mathrm{c}}\left(U^{\prime}\right)$ fixes a neighbourhood of $C_{3}$, an element $\phi \in \operatorname{Stab}\left(C_{1}\right) \subset \operatorname{Symp}_{\mathrm{c}}\left(U^{\prime}\right)$ will induce a symplectomorphism $\bar{\phi}$ of $C_{1}$ which is compactly supported away from the point $\infty=([0: 1],[1$ : $0]) \in C_{1} \cap C_{3}$. The map $\phi \mapsto \bar{\phi}$ gives a fibration

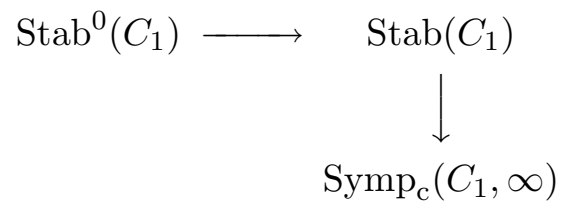

where $\operatorname{Stab}^{0}\left(C_{1}\right)$ is the group of symplectomorphisms $\phi \in \operatorname{Symp}_{\mathrm{c}}\left(U^{\prime}\right)$ which fix $C_{1}$ pointwise and $\operatorname{Symp}_{\mathrm{c}}\left(C_{1}, \infty\right)$ denotes the group of compactly supported symplectomorphisms of $C_{1} \backslash\{\infty\}$. This latter group is contractible, so it suffices to prove that $\operatorname{Stab}^{0}\left(C_{1}\right)$ is weakly homotopy equivalent to $\operatorname{Symp}_{\mathrm{c}}(U)$.

Define $\mathcal{G}$ to be the group of symplectic gauge transformations of the normal bundle to $C_{1}$ in $X$ which equal the identity on some neighbourhood of $\infty \in C_{1}$. It follows from Section 4.1 that this group is weakly contractible. The map $\operatorname{Stab}^{0}\left(C_{1}\right) \rightarrow \mathcal{G}$, which takes a compactly supported symplectomorphism of $U^{\prime}$ fixing $C_{1}$ to its derivative on the normal bundle to $C_{1}$, defines a fibration. Weak contractibility of $\mathcal{G}$ implies that the kernel, $\operatorname{Stab}^{1}\left(C_{1}\right)$, of this fibration is weakly homotopy equivalent to $\operatorname{Stab}^{0}\left(C_{1}\right)$.

This kernel is the group of compactly supported symplectomorphisms of $U^{\prime}$ which fix $C_{1}$ and act trivially on its normal bundle. By the symplectic neighbourhood theorem, this is weakly homotopy equivalent to the group of compactly supported symplectomorphisms of $U=U^{\prime} \backslash C_{1}$.

Putting all this together, weak contractibility of $\operatorname{Symp}_{c}(U)$ follows from the long exact sequence of the fibration

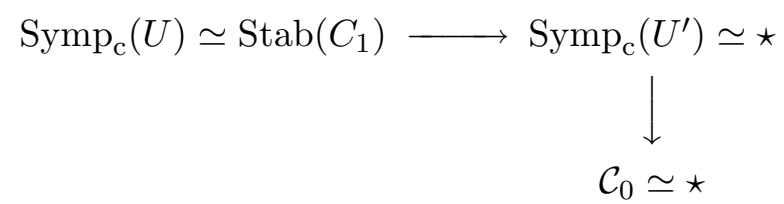

We noted earlier that $\operatorname{Symp}_{\mathrm{c}}(U) \simeq \operatorname{Symp}_{\mathrm{c}}\left(\mathbb{C}^{*} \times \mathbb{C}\right)$, so this proves Theorem 1.6. 
5.2. Proof of Proposition 5.1. To prove weak contractibility of the space of standard configurations, we will need some preliminary work.

5.2.1. Gompf isotopy. We first introduce another space of configurations of spheres.

Definition 5.2. A non-standard configuration in $X$ is an embedded symplectic sphere $S$ satisfying the following properties:

- $S$ is homologous to $C_{1}$,

- $S$ is disjoint from $C_{2}$,

- $S$ intersects $C_{3}$ transversely once at $([0: 1],[1: 0])$,

- there exists a $J \in \mathcal{J}$ making $S, C_{2}$ and $C_{3}$ simultaneously J-holomorphic,

Let $\mathcal{C}$ denote the space of non-standard configurations, topologized as a quotient

$$
\operatorname{Map}\left(\mathbb{C P}^{1}, X\right) / \operatorname{Diff}\left(\mathbb{C P}^{1}\right),
$$

where $\operatorname{Map}\left(\mathbb{C P}^{1}, X\right)$ and $\operatorname{Diff}\left(\mathbb{C P}^{1}\right)$ are given the $\mathcal{C}^{\infty}$-topology.

These are just like standard configurations but without the requirement that they intersect $C_{3}$ in a standard way. An additional subtlety arises from requiring there exists a $J$ making $S$ and $C_{3}$ simultaneously holomorphic: it is not even true that two transverse symplectic linear subspaces of a symplectic vector space can be made simultaneously $J$-holomorphic. This is discussed further below.

There is an inclusion $\iota: \mathcal{C}_{0} \hookrightarrow \mathcal{C}$.

Lemma 5.3. $\iota$ is a weak homotopy equivalence.

The proof of this uses a construction due to Gompf. Specifically, the proof of Lemma 2.3 from [Gom95] implies the following:

Lemma 5.4. Let $N$ be a symplectic two-manifold and $E \rightarrow N$ be a (discsubbundle of a) rank-2 vector bundle with a symplectic form $\omega$ on the total space making the zero-section both symplectic and symplectically orthogonal to the fibres. Let $M$ be a two-dimensional symplectic submanifold of $E$, closed as a subset of $E$, intersecting $N$ transversely at a single point $x$. Then there is a symplectic isotopy $M(t)$ of $M$ for which $M(0)=M, M(t)=M$ outside a small neighbourhood of $M(t) \cap N$ and $M(1)$ agrees with the fibre $E_{x}$ in a small neighbourhood of $x$.

The isotopy defined in that proof depends on: a choice of neighbourhood of $N$ in which $M$ intersects $N$ once transversely and three auxiliary real parameters. Other choices (such as a cut-off function $\mu$ ) can be made to depend only on these real parameters. It is clear from the definitions of these parameters that they can be chosen to depend continuously on $M$ as 
$M$ varies in the space of symplectic submanifolds with the $\mathcal{C}^{\infty}$-topology, and the same neighbourhood of $N$ can be used for $M_{1}$ and $M_{2}$ which are close in the $\mathcal{C}^{\infty}$-topology. We will call the isotopy defined in that proof a Gompf isotopy.

It also follows from Gompf's construction that Gompf isotopy preserves the space $\mathcal{C}$. The only subtlety is that $\mathcal{C}$ consists of configurations whose components can be made simultaneously $J$-holomorphic. One must check that at the intersection points, the tangent planes of the intersecting components can be made simultaneously $J$-holomorphic. In a Darboux chart $\left(\cong \mathbb{R}^{2} \times \mathbb{R}^{2}\right)$ around the intersection point, where one of the intersecting components is mapped to the $\mathbb{R}^{2} \times\{\star\}$ coordinate plane, transversality of the intersection implies one can write the other component as a graph of a map $f: \mathbb{R}^{2} \rightarrow \mathbb{R}^{2}$. The condition that this graph is symplectic is just that $\operatorname{det}\left(f_{\star}\right)>-1$. The condition that the graph can be made simultaneously $J$-holomorphic with $\mathbb{R}^{2} \times\{\star\}$ is that $\operatorname{det}\left(f_{\star}\right) \geq 0$. In the local model used to define it, Gompf isotopy changes $\operatorname{det}\left(f_{\star}\right)$ monotonically towards 0 , so it preserves $\mathcal{C}$.

Proof of Lemma 5.3. Let $f_{1}, f_{2}:\left(S^{n}, \star\right) \rightarrow\left(\mathcal{C}_{0}, C\right)$ represent homotopy classes $\left[f_{1}\right],\left[f_{2}\right]$ in $\pi_{n}\left(\mathcal{C}_{0}, C\right)$. We first show that $\iota_{\star}\left[f_{1}\right]=\iota_{\star}\left[f_{2}\right]$ only if $\left[f_{1}\right]=\left[f_{2}\right]$. Suppose $S:\left(S^{n} \times[1,2], \star\right) \rightarrow(\mathcal{C}, C)$ is a homotopy between $\iota \circ f_{1}$ and $\iota \circ f_{2}$.

By the symplectic neighbourhood theorem there is a neighbourhood $\nu$ of $C_{3}$, isomorphic to a disc-subbundle of $C_{3} \times \mathbb{C}$ and such that $S(x, t) \cap \nu$ satisfies the hypotheses of Lemma 5.4. Pick the parameters small enough to define a Gompf isotopy $\Gamma$ for all spheres $S(x, t)$. Then

$$
\Gamma:\left(S^{n} \times[1,2] \times[1,2], \star\right) \rightarrow(\mathcal{C}, C)
$$

gives a homotopy from $S$ to $S^{\prime}=\Gamma(\cdot, \cdot, 2)$. This new homotopy lies entirely in $\mathcal{C}_{0}$. Since the original maps $f_{i}: S^{n} \rightarrow \mathcal{C}_{0}$ landed in the space of standard configurations and Gompf isotopy preserves the property of being a standard configuration, $S^{\prime}$ is indeed a homotopy connecting $f_{1}$ and $f_{2}$ in $\mathcal{C}_{0}$. This proves injectivity of $\iota_{\star}$.

To prove surjectivity, let $f:\left(S^{n}, \star\right) \rightarrow(\mathcal{C}, C)$ represent a homotopy class $[f]$. For some neighbourhood $\nu$ of $C_{3}$ and sufficiently small choice of parameters one obtains a Gompf isotopy rel $C$ of the image of $f$ into $\mathcal{C}_{0}$. This gives a homotopy class $\left[f^{\prime}\right]$ in $\pi_{n}\left(\mathcal{C}_{0}, C\right)$ and the Gompf isotopy is a homotopy between $\iota_{\star}\left[f^{\prime}\right]$ and $[f]$.

Since $\iota_{\star}$ is an isomorphism on homotopy groups, $\iota$ is a weak homotopy equivalence.

5.2.2. Structures making a configuration holomorphic. Let $S=$ $\bigcup_{i=1}^{n} S_{i}$ be a union of embedded symplectic two-spheres in a symplectic four-manifold $X$. Suppose that the various components intersect transversely 
and that there are no triple intersections. Suppose further that there is an $\omega$-compatible $J$ for which all the components $S_{i}$ are $J$-holomorphic. Let $\mathcal{H}(S)$ denote the space of $\omega$-compatible almost complex structures $J$ for which all components of $S$ are simultaneously $J$-holomorphic.

Lemma 5.5. If the components of $S$ intersect one another symplectically orthogonally then the space $\mathcal{H}(S)$ is weakly contractible.

This lemma is proved in Appendix 8.

5.2.3. Gromov's theory of pseudoholomorphic curves. The following theorem follows from Gromov's theory of pseudoholomorphic curves in symplectic four-manifolds.

Theorem 5.1 ([Gro85]). Given an $\omega$-compatible almost complex structure $J$ on $X=\mathbb{C P}^{1} \times \mathbb{C P}^{1}$ there is a unique $J$-holomorphic curve through ([0 : $1],[1: 0])$ in the homology class $\left[C_{1}\right]$.

If we restrict to the space $\mathcal{H}\left(C^{\prime}\right)$ of almost complex structures which make $C_{2}$ and $C_{3}$ into $J$-holomorphic spheres then this gives us a map

$$
G: \mathcal{H}\left(C^{\prime}\right) \rightarrow \mathcal{C}
$$

since by positivity of intersections, the unique $J$-holomorphic curve through ([0:1], $[1: 0])$ in the homology class $C_{1}$ is then a non-standard configuration, i.e., it cannot intersect $C_{2}$ and it must intersect $C_{3}$ transversely once.

5.2.4. Proof of Proposition 5.1. Let $f:\left(S^{n}, \star\right) \rightarrow\left(\mathcal{C}_{0}, C\right)$ be a based map. We must show that $f$ is nullhomotopic. Let $\infty \in S^{n}$ be the antipode to $\star$ and let $R$ be the space of great half-circles $r(t)$ connecting $r(0)=\star$ to $r(1)=\infty$. Each such half-circle defines an isotopy $f(r(t))$ of standard configurations. This isotopy extends to a small neighbourhood of the standard configurations by the symplectic neighbourhood theorem and then to a global isotopy $\psi_{r, t} \in \operatorname{Symp}(C)$ by Banyaga's isotopy extension theorem (since $H^{2}\left(X, S \cup C_{3} ; \mathbb{R}\right)=0$ for all the standard configurations). We can assume this isotopy fixes a neighbourhood of $C_{2}$ and $C_{3}$ pointwise.

Suppose $J_{0}$ is an $\omega$-compatible almost complex structure for which the basepoint $C \in \mathcal{C}_{0}$ is $J_{0}$-holomorphic. Then the standard configuration $f(r(t))$ is $\left(\psi_{r, t}\right)_{\star} J_{0}$-holomorphic. Let $\bar{B}^{n}=S^{n} \backslash\{\infty\} \cup S^{n-1}$ be the compactification of $S^{n} \backslash\{\infty\}$ which adds an endpoint to every open half-circle $\{r(t)\}_{t \in[0,1]}$ (this is the oriented real blow-up of $S^{n}$ at $\infty$ ). Define a map

$$
\begin{gathered}
\tilde{f}_{1}: \bar{B}^{n} \rightarrow \mathcal{H}\left(C^{\prime}\right), \\
\tilde{f}_{1}(r, t)=\left(\psi_{r, t}\right)_{\star} J_{0} \in \mathcal{H}\left(C^{\prime}\right),
\end{gathered}
$$


which lifts $\bar{B}^{n} \rightarrow S^{n} \stackrel{f}{\rightarrow} \mathcal{C}_{0}$ (where the first map collapses $S^{n-1}$ to the point $\infty)$.

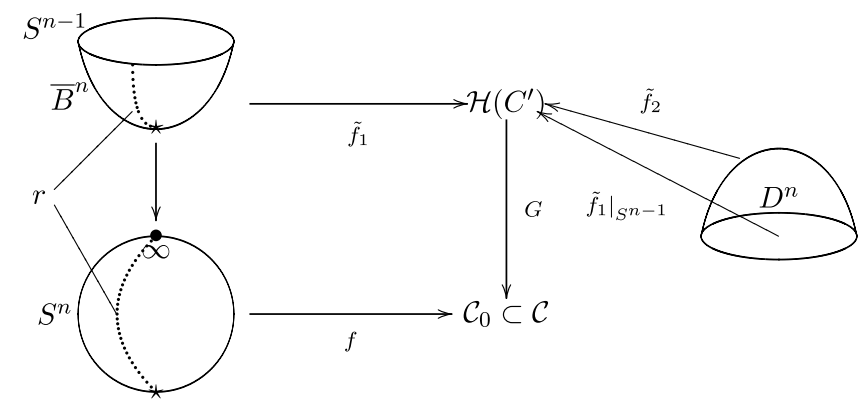

The image of the restriction $\left.\tilde{f}_{1}\right|_{S^{n-1}}: S^{n-1} \rightarrow \mathcal{H}\left(C^{\prime}\right)$ consists of $\omega$-compatible almost complex structures which make the standard configuration $f(\infty)$ holomorphic. The space of such almost complex structures $\left(\mathcal{H}(f(\infty)) \subset \mathcal{H}\left(C^{\prime}\right)\right)$ is contractible by Lemma 5.5 and hence there is a map $\tilde{f}_{2}: D^{n} \rightarrow \mathcal{H}\left(C^{\prime}\right)$ with $\left.\tilde{f}_{2}\right|_{\partial D^{n}}=\left.\tilde{f}\right|_{S^{n-1}}$ (where $D^{n}$ is the closed $n$-ball) such that $G \circ \tilde{f}_{2}(x)=f(\infty)$. Glue $\tilde{f}_{1}$ and $\tilde{f}_{2}$ to obtain a map $\tilde{f}=\tilde{f}_{1} \cup \tilde{f}_{2}: S^{n} \rightarrow \mathcal{H}\left(C^{\prime}\right)$ for which $G \circ \tilde{f}$ is homotopic to $f$.

By Lemma $5.5 \mathcal{H}\left(C^{\prime}\right)$ is contractible, $\tilde{f}$ is nullhomotopic via a map $H$ : $D^{n+1} \rightarrow \mathcal{H}\left(C^{\prime}\right)$. Therefore $G \circ H$ is a nullhomotopy of $f$ in $\mathcal{C}$. But we have observed (Lemma 5.3) that $\mathcal{C}_{0} \rightarrow \mathcal{C}$ is a weak homotopy equivalence, hence $f$ is nullhomotopic in $\mathcal{C}_{0}$.

\section{Del Pezzo surfaces}

6.1. Results. In this section, we use known results on symplectomorphism groups of affine varieties to calculate the weak homotopy type of the symplectomorphism groups of some Del Pezzo surfaces. We recall:

Definition 6.1. A symplectic del Pezzo surface is the symplectic manifold underlying a smooth complex projective surface with ample anticanonical line bundle. Equivalently it is one of the following symplectic four-manifolds:

- $Q=S^{2} \times S^{2}$ equipped with the product of the area-1 Fubini-Study forms on each factor (this also arises as the Kähler form on a smooth quadric hypersurface in $\mathbb{C P}^{3}$ ),

- A symplectic blow-up $\mathbb{D}_{n}$ of $\mathbb{C P}^{2}$ (with its anticanonical symplectic form $\left.3 \omega_{\mathrm{FS}}\right)$ in $n<9$ symplectic balls of equal volume such that

$$
\omega_{\mathbb{D}_{n}}\left(E_{k}\right)=\omega_{\mathrm{FS}}(H),
$$

where $E_{k}$ is an exceptional sphere and $H$ is a line in $\mathbb{C P}^{2}$.

We prove the following. 
Theorem 1.3. Let $\operatorname{Symp}_{0}(X)$ denote the group of symplectomorphisms of $X$ acting trivially on homology and let $\operatorname{Diff}^{+}\left(S^{2}, 5\right)$ denote the group of diffeomorphisms of $S^{2}$ fixing five points.

- $\operatorname{Symp}\left(\mathbb{D}_{3}\right) \simeq T^{2}$,

- $\operatorname{Symp}\left(\mathbb{D}_{4}\right) \simeq \star$,

- $\operatorname{Symp}\left(\mathbb{D}_{5}\right) \simeq \operatorname{Diff}^{+}\left(S^{2}, 5\right)$,

where all $\simeq$ signs denote weak homotopy equivalence.

The third result in the theorem deserves some comment. Seidel has shown [Sei08] that there are maps $C / G \rightarrow B \operatorname{Symp}\left(\mathbb{D}_{5}\right) \rightarrow C / G$ whose composition is homotopic to the identity. Here $C$ is the configuration space of five ordered points on $S^{2}$ and $G$ is the group $\mathbb{P} S L_{2} \mathbb{C}$ of Möbius transformations of $S^{2}$. $G$ acts freely on $C$, so we get a principal $G$-bundle:

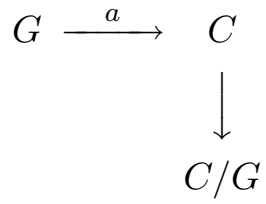

The group $\operatorname{Diff}^{+}\left(S^{2}\right)$ also acts on $C$ and there is an evaluation fibration

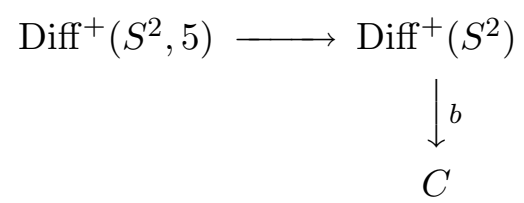

The maps $a$ and $b$ are actually homotopy equivalent (in view of the fact that the inclusion $G \rightarrow \operatorname{Diff}^{+}\left(S^{2}\right)$ is a homotopy equivalence), so $C / G$ is weakly homotopy equivalent to $B \operatorname{Diff}^{+}\left(S^{2}, 5\right)$. In fact, Teichmüller theory shows that $\operatorname{Diff}^{+}\left(S^{2}, 5\right)$ is weakly homotopy equivalent to its group $B$ of components. We can find the group $B$ as follows. $\pi_{1}(C)$ is the group $\operatorname{PBr}\left(S^{2}, 5\right)$ of five-strand pure braids on the sphere. $\pi_{1}(G)$ is $\mathbb{Z} / 2$, generated by a loop of rotations through 0 to $2 \pi$. By the homotopy long exact sequence associated to the fibration $G \rightarrow C \rightarrow C / G, \pi_{1}(C / G)$ is the cokernel of the map $\mathbb{Z} / 2 \rightarrow P \operatorname{Br}\left(S^{2}, 5\right)$. It is not hard to see that the image of this map is the full twist $\tau$ (which has order 2 in the braid group), so $B \cong P \operatorname{Br}\left(S^{2}, 5\right) /\langle\tau\rangle$.

6.2. Outline of proof. The proofs in all three cases run along similar lines. We outline the general picture before filling in details individually. Therefore let $(X, \omega)$ denote any of $\mathbb{D}_{3}, \mathbb{D}_{4}$ or $\mathbb{D}_{5}$, let $\mathcal{J}$ denote the space of $\omega$-tame almost complex structures on $X$ and let $\operatorname{Symp}_{0}(X)$ denote the group of symplectomorphisms acting trivially on $H^{*}(X, \mathbb{Z})$.

In each case we will identify a divisor $C=\bigcup_{i} C_{i} \subset X$ such that

- $C$ consists of embedded $J$-holomorphic -1 -spheres which intersect one another symplectically orthogonally,

- $H^{2}(X, C ; \mathbb{R})=0$. 
Definition 6.2. A standard configuration in $X$ will mean a configuration $S=\bigcup_{i} S_{i}$ of embedded symplectic spheres such that

- $\left[S_{i}\right]=\left[C_{i}\right]$ for all $i$,

- there exists a $J \in \mathcal{J}$ simultaneously making every component $S_{i}$ into a $J$-holomorphic sphere,

- at every intersection point of the configuration, the components intersect $\omega$-orthogonally.

Let $\mathcal{C}_{0}$ denote the space of standard configurations.

Proposition 6.1. $\mathcal{C}_{0}$ is weakly contractible.

Proof. The proof of this proposition is very similar to the proof of Proposition 5.1. The Gompf isotopy argument is slightly more involved, as there are several components that need to be isotoped at once. The crucial input from Gromov's theory of pseudoholomorphic curves is the following:

Lemma 6.1 ([Pin08], Lemma 1.2). Let $(M, \omega)$ be a symplectic fourmanifold not diffeomorphic to $\mathbb{C P}^{2} \# \overline{\mathbb{C P}^{2}}$. Then, for any choice of $\omega$-tame almost complex structure $J$, all symplectic exceptional classes of minimal symplectic area are represented by an embedded J-holomorphic sphere.

In particular, any $S_{1}, S_{2} \in \mathcal{C}_{0}$ are isotopic through standard configurations. The property that the configurations are symplectically orthogonal where they intersect and the condition $H^{2}(X, C ; \mathbb{R})=0$ allow us to extend such an isotopy to a global symplectomorphism of $X$ by Banyaga's theorem. Therefore:

Proposition 6.2. $\operatorname{Symp}_{0}(X)$ acts transitively on $\mathcal{C}_{0}$.

Banyaga's theorem now gives us a fibration:

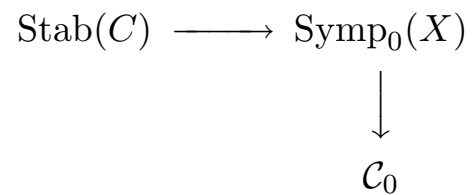

so the group $\operatorname{Stab}(C)$ of symplectomorphisms of $X$ which fix the configuration $C$ setwise (and act trivially on the set of components) is weakly homotopy equivalent to $\operatorname{Symp}_{0}(X)$ (by the homotopy long exact sequence, since $\mathcal{C}_{0}$ is weakly contractible).

The next step is to investigate $\operatorname{Stab}(C)$. An element $\phi \in \operatorname{Stab}(C)$ restricts to give an element $\left(\left.\phi\right|_{C_{1}}, \ldots,\left.\phi\right|_{C_{n}}\right) \in \operatorname{Symp}(C)$ (see Section 4.3). Working in a neighbourhood of $C$, pulling back Hamiltonians to the normal bundles and cutting them off outside a certain radius, one can prove homotopy-lifting for this restriction map (see for example the proof of Proposition 7.2 later). 
Therefore the restriction map fits into a fibration:

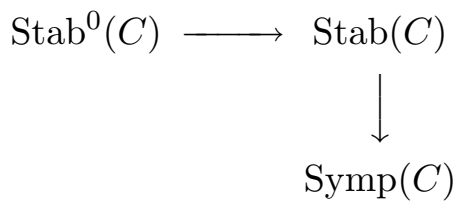

where $\operatorname{Stab}^{0}(C)$ is the group of symplectomorphisms of $X$ which fix $C$ pointwise.

As explained in Section 4.2, the group $\operatorname{Symp}(C)$ can be understood purely in terms of $\operatorname{Diff}^{+}(C)$, thanks to Moser's theorem, and thereby reduced to a problem in Teichmüller theory. To understand the stabilizer $\operatorname{Stab}^{0}(C)$, we need to consider the fibration:

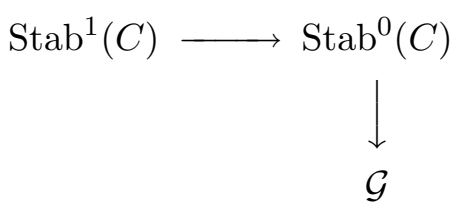

which takes a symplectomorphism fixing $C$ to the induced map on the normal bundles of $C$ (see Section 4.1). Here $\mathcal{G}$ is the group of symplectic gauge transformations of the normal bundles to components of $C$ and $\operatorname{Stab}^{1}(C)$ consists of those symplectomorphisms fixing $C$ pointwise and acting trivially on the normal bundles of its components. By the symplectic neighbourhood theorem:

Lemma 6.2. $\operatorname{Stab}^{1}(C)$ is weakly homotopy equivalent to the group of compactly supported symplectomorphisms of $U=X \backslash C$.

In all our cases we will understand $\operatorname{Symp}_{\mathrm{c}}(U)$ and $\mathcal{G}$. The homotopy exact sequences of the various fibrations (pictured below) will allow us to work backward, finding the homotopy groups of $\operatorname{Stab}^{0}(C)$ and $\operatorname{Stab}(C) \simeq$ $\operatorname{Symp}_{0}(X)$.

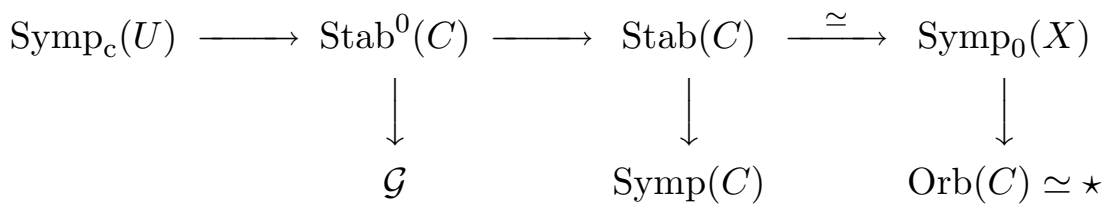

6.3. The case $\mathbb{D}_{3}$. Here we consider the configuration of exceptional curves in the homology classes $S_{12}=H-E_{1}-E_{2}, S_{13}=H-E_{1}-E_{3}, S_{23}=$ $H-E_{2}-E_{3}, E_{1}$ and $E_{2}$. If we included $E_{3}$, these would form a hexagonal 
configuration, but in order to ensure $H^{2}(X, C ; \mathbb{R})=0$, we need to omit $E_{3}$.

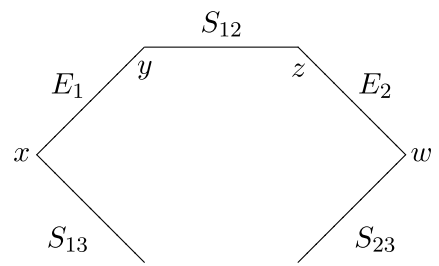

The complement of $U=X \backslash C$ is biholomorphic to $\mathbb{C} \times \mathbb{C}^{*}$ and $\omega$ restricted to $U$ is $-d d^{c} h$ for a plurisubharmonic function $h$ on $U$. By Proposition $2.2, \operatorname{Symp}_{\mathrm{c}}(U) \simeq \operatorname{Symp}_{\mathrm{c}}\left(\mathbb{C} \times \mathbb{C}^{*}\right)$. By Theorem 1.6, Symp $\left(\mathbb{C} \times \mathbb{C}^{*}\right)$ is contractible, so $\operatorname{Stab}^{0}(C) \simeq \mathcal{G}$. Let us calculate the groups $\mathcal{G}$ and $\operatorname{Symp}(C)$ :

- $\mathcal{G}\left(S_{13}\right) \cong \mathcal{G}\left(S_{23}\right) \cong \mathcal{G}_{1} \simeq \star$,

- $\mathcal{D}\left(S_{13}\right) \cong \mathcal{D}\left(S_{23}\right) \cong \mathcal{D}_{1} \simeq S^{1}$

- $\mathcal{G}\left(S_{12}\right) \cong \mathcal{G}\left(E_{1}\right) \cong \mathcal{G}\left(E_{2}\right) \cong \mathcal{G}_{2} \simeq \mathbb{Z}$

- $\mathcal{D}\left(S_{12}\right) \cong \mathcal{D}\left(E_{1}\right) \cong \mathcal{D}\left(E_{2}\right) \cong \mathcal{D}_{2} \simeq S^{1}$,

therefore $\mathcal{G} \simeq \mathbb{Z}^{3}$ and $\operatorname{Symp}(C) \simeq\left(S^{1}\right)^{5}$. Since $\operatorname{Stab}^{0}(C) \simeq \mathcal{G}$ and $\operatorname{Stab}(C) \simeq$ $\operatorname{Symp}\left(\mathbb{D}_{3}\right)$, the fibration $(*)$ yields the long exact sequence:

$$
1 \rightarrow \pi_{1}\left(\operatorname{Symp}\left(\mathbb{D}_{3}\right)\right) \rightarrow \mathbb{Z}^{5} \rightarrow \mathbb{Z}^{3} \rightarrow \pi_{0}\left(\operatorname{Symp}\left(\mathbb{D}_{3}\right)\right) \rightarrow 1 .
$$

The calculation reduces to understanding the map $\mathbb{Z}^{5} \rightarrow \mathbb{Z}^{3}$. This comes from a map $\pi_{1}(\operatorname{Symp}(C)) \rightarrow \pi_{0}(\mathcal{G})$, so this can actually be understood purely by considering a neighbourhood of $C$ as explained in Section 4.3. Let us pick a basis for $\pi_{1}(\operatorname{Symp}(C))$. Let $\operatorname{rot}\left(C_{j}\right)$ denote the element represented by the loops $\phi_{2 \pi}$ defined in Lemma 4.1, i.e., the loop of sympletomorphisms which rotate $C_{j}$ through $2 \pi$ and leave the other components fixed. These generate the group $\pi_{1}(\operatorname{Symp}(C))$.

By Lemma 4.1 , the map $\mathbb{Z}^{5} \rightarrow \mathbb{Z}^{3}$ is:

$$
\begin{aligned}
\operatorname{rot}\left(S_{13}\right) & \mapsto g_{E_{1}}(x) \in \pi_{0}\left(\mathcal{G}\left(E_{1}\right)\right), \\
\operatorname{rot}\left(S_{23}\right) & \mapsto g_{E_{2}}(w) \quad \in \pi_{0}\left(\mathcal{G}\left(E_{2}\right)\right)
\end{aligned}
$$

and

$$
\begin{array}{rlll}
\operatorname{rot}\left(E_{1}\right) & \mapsto\left(0, g_{S_{13}}(x)\right) & \in \pi_{0}\left(\mathcal{G}\left(S_{13}\right)\right) \times \pi_{0}\left(\mathcal{G}\left(S_{12}\right),\right. \\
\operatorname{rot}\left(E_{2}\right) & \mapsto\left(g_{S_{12}}(z), 0\right) & \in \pi_{0}\left(\mathcal{G}\left(S_{12}\right)\right) \times \pi_{0}\left(\mathcal{G}\left(S_{23}\right)\right), \\
\operatorname{rot}\left(S_{12}\right) & \mapsto\left(g_{E_{1}}(y), g_{E_{2}}(z)\right) & \in \pi_{0}\left(\mathcal{G}\left(E_{1}\right)\right) \times \pi_{0}\left(\mathcal{G}\left(E_{2}\right)\right)
\end{array}
$$

Therefore the map is surjective with kernel of rank 2 . This implies

$$
\pi_{1}\left(\operatorname{Symp}_{0}\left(\mathbb{D}_{3}\right)\right)=\mathbb{Z}^{2}, \pi_{0}\left(\operatorname{Symp}_{0}\left(\mathbb{D}_{3}\right)\right)=0
$$

as stated. 
6.4. The case $\mathbb{D}_{4}$. The configuration $C$ consists of exceptional spheres in the homology classes $S_{12}=H-E_{1}-E_{2}, S_{34}=H-E_{3}-E_{4}, E_{1}, E_{2}, E_{3}$ and $E_{4}$.

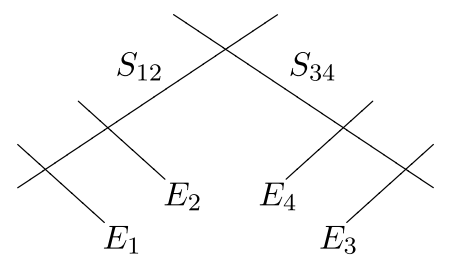

Let us calculate the homotopy types of $\mathcal{G}$ and $\operatorname{Symp}(C)$ :

- $\mathcal{G}\left(S_{12}\right) \cong \mathcal{G}\left(S_{34}\right) \cong \mathcal{G}_{3} \simeq \mathbb{Z}^{2}$,

- $\mathcal{D}\left(S_{12}\right)=\mathcal{D}\left(S_{34}\right)=\mathcal{D}_{3} \simeq \star$,

- $\mathcal{G}\left(E_{1}\right) \cong \cdots \cong \mathcal{G}\left(E_{4}\right) \cong \mathcal{G}_{1} \simeq \star$,

- $\mathcal{D}\left(E_{1}\right) \cong \ldots \cong \mathcal{D}\left(E_{4}\right)=\mathcal{D}_{1} \simeq S^{1}$,

Therefore $\mathcal{G} \simeq \mathbb{Z}^{4}$ and $\operatorname{Symp}(C) \simeq\left(S^{1}\right)^{4}$.

The complement $U=X \backslash C$ is biholomorphic to $\mathbb{C}^{2}$ and the restriction of $\omega$ to $U$ arises as $-d d^{\mathrm{c}} h$ for a plurisubharmonic function $h$ on $U$, so by Proposition 2.2 $\operatorname{Symp}_{\mathrm{c}}(U) \simeq \operatorname{Symp}_{\mathrm{c}}\left(\mathbb{C}^{2}\right)$. Gromov has shown $\operatorname{Symp}_{\mathrm{c}}\left(\mathbb{C}^{2}\right) \simeq$ $\star$. This simplifies the fibrations:

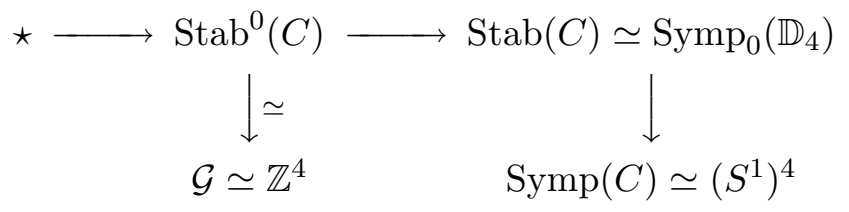

We therefore get a long exact sequences of homotopy groups:

$$
1 \rightarrow \pi_{1}\left(\operatorname{Symp}_{0}\left(\mathbb{D}_{4}\right)\right) \rightarrow \mathbb{Z}^{4} \rightarrow \mathbb{Z}^{4} \rightarrow \pi_{0}\left(\operatorname{Symp}_{0}\left(\mathbb{D}_{4}\right)\right) \rightarrow 1 .
$$

In the notation introduced in the previous section, the map $\mathbb{Z}^{4} \rightarrow \mathbb{Z}^{4}$ is given by

$$
\operatorname{rot}\left(E_{i}, x_{i}\right) \mapsto g_{S_{i k}}\left(x_{i}\right) \in \pi_{0}\left(\mathcal{G}\left(S_{i k}\right)\right)
$$

so it is an isomorphism and all the homotopy groups of $\operatorname{Symp}_{0}\left(\mathbb{D}_{4}\right)$ vanish.

6.5. The case $\mathbb{D}_{\mathbf{5}}$. The relevant configuration $C$ is the total transform of the conic through the five blow-up points, i.e., the exceptional curves in homology classes $E_{1}, \ldots, E_{5}$ and $Q=2 H-\sum_{i=1}^{5} E_{i}$.

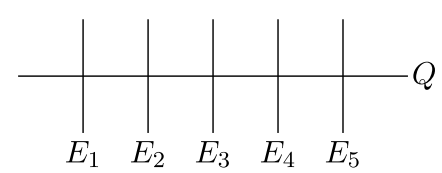


Let us calculate the homotopy types of $\mathcal{G}$ and $\operatorname{Symp}(C)$ :

- $\mathcal{G}(Q) \cong \mathcal{G}_{5} \simeq \mathbb{Z}^{4}$,

- $\mathcal{D}(Q) \cong \operatorname{Diff}\left(5, S^{2}\right)$

- $\mathcal{G}\left(E_{1}\right) \cong \cdots \cong \mathcal{G}\left(E_{5}\right) \cong \mathcal{G}_{1} \simeq \star$,

- $\mathcal{D}\left(E_{1}\right) \cong \cdots \cong \mathcal{D}\left(E_{5}\right) \cong \mathcal{D}_{1} \simeq S^{1}$, so $\mathcal{G} \simeq \mathbb{Z}^{4}$ and $\operatorname{Symp}(C) \simeq \operatorname{Diff}\left(5, S^{2}\right) \times\left(S^{1}\right)^{5}$.

The complement $U=X \backslash C$ is biholomorphic to the complement of a conic in $\mathbb{C P}^{2}$ and the restriction of $\omega$ to $U$ is $-d d^{c} h$ for a plurisubharmonic function $h$, so by Proposition 2.2 $\operatorname{Symp}_{\mathrm{c}}(U) \simeq \operatorname{Symp}_{\mathrm{c}}\left(T^{*} \mathbb{R P}^{2}\right)$. By Section $3, \operatorname{Symp}_{\mathrm{c}}\left(T^{*} \mathbb{R} \mathbb{P}^{2}\right) \simeq \mathbb{Z}$. The fibrations become

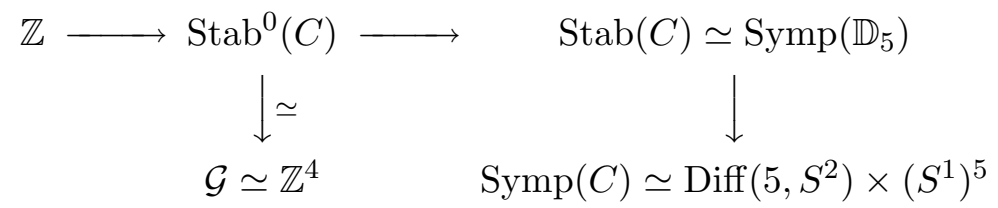

From this we deduce that $\operatorname{Stab}^{0}(C)$ is weakly equivalent to a discrete group $Z$ which is an extension of $\mathbb{Z}^{4}$ by $\mathbb{Z}$ and that the relevant long exact sequence of homotopy groups is

$$
1 \rightarrow \pi_{1}\left(\operatorname{Symp}\left(\mathbb{D}_{5}\right)\right) \rightarrow \mathbb{Z}^{5} \stackrel{\psi}{\rightarrow} Z \rightarrow \pi_{0}\left(\operatorname{Symp}\left(\mathbb{D}_{5}\right)\right) \rightarrow B \rightarrow 1,
$$

where $B$ is the group $\operatorname{PBr}\left(5, S^{2}\right) /\langle\tau\rangle$ of components of $\operatorname{Diff}\left(5, S^{2}\right)$.

The key, then, is to understand this map $\psi: \mathbb{Z}^{5} \rightarrow Z$. The composition of this map with $\pi_{0}\left(\operatorname{Stab}^{0}(C)\right) \rightarrow \mathcal{G} \cong \mathbb{Z}^{4}$ is surjective by Lemma 4.1 (the preimage of $g_{Q}\left(x_{i}\right) \in \pi_{0}(\mathcal{G}(Q))$ is $\left.\operatorname{rot}\left(E_{i}, x_{i}\right)\right)$. It remains to show that if $\chi$ is the isotopy class of the Dehn twist in the Lagrangian $\mathbb{R P}^{2} \subset U$ then $\chi=\psi(\eta)$ for some $\eta \in \pi_{1}(\operatorname{Symp}(C))$.

We begin with a lemma whose proof is analogous to that of Lemma 1.8 from [Sei08] (which is the same statement for Lagrangian spheres):

Lemma 6.3. Let $L \subset X$ be a Lagrangian $\mathbb{R P}^{2}, \mho$ a Weinstein neighbourhood of $L$ and suppose there is a Hamiltonian circle action on $X \backslash L$ which commutes with the round cogeodesic flow on $\mho \backslash L$. Then the Dehn twist in $L$ is symplectically isotopic to $i d$.

Let $\mu$ be the moment map for the $\mathrm{SO}(3)$-action on $T^{*} \mathbb{R P}^{2}$. Then $\|\mu\|$ generates a Hamiltonian circle action on $T^{*} \mathbb{R P}^{2} \backslash \mathbb{R P}^{2}$ which commutes with the round cogeodesic flow. Symplectically cutting along a level set of $\|\mu\|$ gives $\mathbb{C P}^{2}$ and the reduced locus is a conic. Pick five points on the conic and $\|\mu\|$ equivariant balls of equal volume centred on them. $\mathbb{D}_{5}$ is symplectomorphic to the blow up in these five balls and the circle action preserves the exceptional locus (the union of the five blow-up spheres and the proper transform of the conic). Hence we have constructed a loop in $\operatorname{Symp}(C)$ which maps to $\chi$. Since the circle action simultaneously rotates the normal bundles to 
the blow-up spheres it should be clear that this element corresponds to the diagonal element $(1, \ldots, 1) \in \mathbb{Z}^{5}$.

This completes the proof of Theorem 1.3.

\section{The $A_{n}$-Milnor fibres}

In this section we prove the following theorem:

Theorem 1.4. Let $W$ be the $A_{n}$-Milnor fibre, the affine variety given by the equation

$$
x^{2}+y^{2}+z^{n}=1,
$$

and let $\omega$ be the Kähler form on $W$ induced from the ambient $\mathbb{C}^{3}$. Then the group of compactly supported symplectomorphisms of $(W, \omega)$ is weakly homotopy equivalent to its group of components. This group of components injects homomorphically into the braid group $\mathrm{Br}_{n}$ of $n$-strands on the disc.

7.1. Compactification. We begin by compactifying $W$ to a projective rational surface. Let $\xi_{k}=\exp (2 \pi \mathrm{i} k / n)$ and denote by $P_{k}(c)$ the polynomial $\frac{c^{n}-1}{c-\xi_{k}}$. Define

- $X$ to be the blow-up of $\mathbb{C P}^{2}$ at the points $\left\{\left[\xi_{k}: 0: 1\right]\right\}_{k=1}^{n}$. This can be thought of as the subvariety of $M=\mathbb{C P}^{2} \times \prod_{k=1}^{n} \mathbb{C P}_{k}^{1}$ given by the equations

$$
a_{k} y=b_{k}\left(x-\xi_{k} z\right) \quad \text { for } k=1, \ldots, n
$$

in coordinates

$$
\left([x: y: z],\left[a_{1}: b_{1}\right], \ldots,\left[a_{n}: b_{n}\right]\right) \text { on } M .
$$

- The pencil of curves $P_{t}$ which are proper transforms of the pencil of lines through $[0: 1: 0]$, parametrized by $t=[x: z]$. In particular, notice that

$$
P_{\infty}=\{([x: y: 0],[x: y], \ldots,[x: y])\} .
$$

- The curve $C_{n+1}=P_{\infty}$.

- The curve $C_{n+2}=\{[x: 0: z]\} \times \prod_{k=1}^{n}\{[1: 0]\}$ which is the proper transform of the line through the blow-up points. This is a section of the pencil $P_{t}$.

- The exceptional spheres $C_{k}(1 \leq k \leq n)$ of the blow-up. $C_{k}$ is given in equations by

$$
\left\{\left[\xi_{k}: 0: 1\right]\right\} \times\{[1: 0]\} \times \cdots \times \mathbb{C P}_{k}^{1} \times \cdots \times\{[1: 0]\}
$$

and constitutes one component of the singular curve $P_{\xi_{k}}$.

- The divisor $C^{\prime}=C_{n+1} \cup C_{n+2}$ and its complement $U^{\prime}=X \backslash C^{\prime}$.

- The divisor $C=C^{\prime} \cup \bigcup_{i=1}^{n} C_{k}$ and its complement $U=X \backslash C$. 


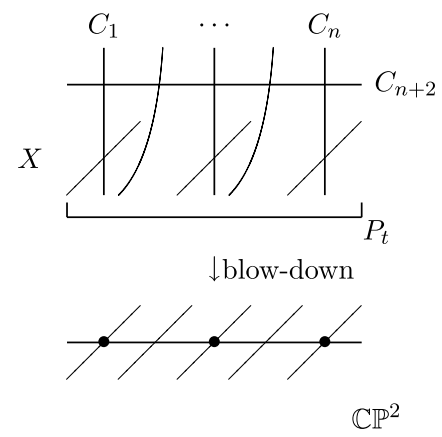

Lemma 7.1. $U^{\prime}$ is biholomorphic to $W$ and $U$ is biholomorphic to $\mathbb{C}^{*} \times \mathbb{C}$.

Proof. We proceed by introducing affine charts on $U^{\prime}$ and writing down explicit partial maps $W \rightarrow U^{\prime}$ which glue compatibly to give a biholomorphism.

Let $\mathbb{C}_{+}, \mathbb{C}_{-}$denote the upper and lower (Zariski) hemispheres of $\mathbb{C P}^{1}$, respectively. For any partition $\sigma$ of $\{1, \ldots, n\}$ into two sets $\sigma_{+}$and $\sigma_{-}$let $( \pm)^{\sigma}(k)$ be \pm 1 if $k \in \sigma_{ \pm}$, respectively, and consider the affine open set

$$
M_{\sigma}=\mathbb{C}^{2} \times \prod_{k=1}^{n} \mathbb{C}_{( \pm)^{\sigma}(k)} \subset M .
$$

Denote by $U_{\sigma}^{\prime}$ the intersection $U^{\prime} \cap M_{\sigma}$. Notice that unless $\left|\sigma_{-}\right| \leq 1, y$ cannot vanish, so

$$
\bigcup_{\left|\sigma_{-}\right| \geq 2} U_{\sigma}^{\prime}=\bigcap_{\left|\sigma_{-}\right| \geq 2} U_{\sigma}^{\prime}=\left\{\left((x, y),\left[x-\xi_{1}: y\right], \ldots,\left[x-\xi_{n}: y\right]\right): y \neq 0\right\} .
$$

We define $\sigma^{k}$ to be the partition with $\sigma_{-}=\{k\}$. Since we have excised $C_{n+2}$, we do not need to consider the partition $\sigma^{\infty}$ with $\sigma_{-}=\emptyset$.

Recall that $W=\left\{(a, b, c) \in \mathbb{C}^{3}: a^{2}+b^{2}+c^{n}=1\right\}$. For each $\sigma$ we define a partial map $\psi_{\sigma}: W \rightarrow U_{\sigma}^{\prime}$ (i.e., a map whose domain is implicitly defined as the region on which the map is well-defined) by

$$
\psi_{\sigma}(a, b, c)=\left((c, a+\mathrm{i} b),\left[c-\xi_{1}: a+\mathrm{i} b\right], \ldots,\left[c-\xi_{n}: a+\mathrm{i} b\right]\right)
$$

for $\left|\sigma_{-}\right| \geq 2$ and

$$
\begin{aligned}
\psi_{\sigma^{k}}(a, b, c)= & \left((c, a+\mathrm{i} b),\left[c-\xi_{1}: a+\mathrm{i} b\right], \ldots,\left[a-\mathrm{i} b: P_{k}(c)\right], \ldots\right. \\
& \left.\times\left[c-\xi_{n}: a+\mathrm{i} b\right]\right) .
\end{aligned}
$$

Since $a^{2}+b^{2}=(a+\mathrm{i} b)(a-\mathrm{i} b)=1-c^{n}$ and $P_{k}(c)\left(c-\xi_{k}\right)=c^{n}-1$, the standard $a_{i} \mapsto 1 / a_{i}$ transition maps for the affine charts on $\mathbb{C P}^{1}$ allow us to glue these partial maps into a global biholomorphism $W \rightarrow U^{\prime}$.

To identify $U$ and $\mathbb{C}^{*} \times \mathbb{C}$, notice that $U$ is just the subset

$$
\left\{\left((x, y),[x-\xi: y], \ldots,\left[x-\xi^{n+1}: y\right]\right): y \neq 0\right\}
$$

from above. 
We now construct a symplectic form on the compactification $X$. Let $\Omega$ denote the symplectic form on $M=\mathbb{C P}^{2} \times \prod_{k=1}^{n} \mathbb{C P}_{k}^{1}$ which is the product of the Fubini-Study forms on each factor, normalized to give a complex line area 1. $X$ inherits a symplectic form $\omega$ from its embedding in $M$ and it is easy to see that

$$
\begin{aligned}
{\left[C_{n+2}\right] } & =\left[C_{n+1}\right]-\sum_{k=1}^{n}\left[C_{k}\right], \\
\omega\left(C_{n+1}\right) & =n+1, \\
\omega\left(C_{n+2}\right) & =1 \\
\omega\left(C_{k}\right) & =1 \\
P D[\omega] & =n\left[C_{n+1}\right]+\left[C_{n+2}\right], \\
c_{1}(X) & =3\left[C_{n+1}\right]-\sum_{k=1}^{n}\left[C_{k}\right] .
\end{aligned}
$$

Moreover, the following observation will prove extremely useful:

Lemma 7.2. At the intersection points $C_{n+2} \cap P_{\infty}$ and $C_{n+2} \cap C_{k}$, the various intersecting components are all $\omega$-symplectically orthogonal. Indeed $C_{n+2}$ is symplectically orthogonal to all the members of the pencil $P_{t}$.

This can be checked by hand. We also notice that since $U$ is the intersection of an affine open subset of $M$ with $X$ that $\left.\omega\right|_{U}$ arises from a plurisubharmonic function. The divisor $n C_{n+1}+C_{n+2}$ satisfies the Nakai-Moishezon criterion for ampleness, so by the first proposition in [GH94, Chapter 1, Section 2], there is also a plurisubharmonic function $\phi_{C^{\prime}}$ on $U^{\prime}$ for which $-d d^{\mathrm{c}} \phi_{C^{\prime}}=\left.\omega\right|_{U^{\prime}}$. By Proposition 2.2, this implies:

Lemma 7.3. $\operatorname{Symp}_{\mathrm{c}}(U) \simeq \operatorname{Symp}_{\mathrm{c}}\left(\mathbb{C}^{*} \times \mathbb{C}\right)$ and $\operatorname{Symp}_{\mathrm{c}}\left(U^{\prime}\right) \simeq \operatorname{Symp}_{\mathrm{c}}(W)$.

7.2. Proof of Theorem 1.4. We now proceed to the proof of Theorem 1.4. As before we introduce the notion of a standard configuration:

Definition 7.1. A standard configuration in $X$ will mean an unordered $n$-tuple of embedded symplectic spheres $\left\{S_{i}\right\}_{i=1}^{n}$ satisfying the following properties:

- each $S_{i}$ is disjoint from $C_{n+1}$,

- $\left[S_{i}\right]=\left[C_{i}\right]$,

- there exists a $J \in \mathcal{J}$ for which all the spheres $S_{i}, C_{n+1}$ and $C_{n+2}$ are $J$-holomorphic.

- there is a neighbourhood $\nu$ of $C_{n+2}$ such that, for all $i \in\{1, \ldots, n\}$,

$$
S_{i} \cap \nu=P_{t_{i}} \cap \nu
$$

where $t_{i}=S_{i} \cap C_{n+2}$. 
Let $\mathcal{C}_{0}$ denote the space of all standard configurations.

Note that we really do want unordered configurations here in order to obtain the full (rather than the pure) braid group.

Proposition 7.1. $\mathcal{C}_{0}$ is weakly contractible.

Proof. The proof of this is similar to the proof of Proposition 5.1. The input from Gromov's theory of pseudoholomorphic curves is Lemma 6.1, mentioned in the proof of Proposition 6.1.

Let $\operatorname{Conf}(n)$ denote the space of configurations of unordered points in $C_{n+2} \backslash\{\infty\}$, where $\infty$ denotes the point of intersection between $C_{n+2}$ and $P_{\infty}$. Define the map

$$
\begin{aligned}
\Psi: \mathcal{C}_{0} & \rightarrow \operatorname{Conf}(n), \\
S=\bigcup_{i=1}^{n} S_{i} & \mapsto\left\{S_{1} \cap C_{n+2}, \ldots, S_{n} \cap C_{n+2}\right\} .
\end{aligned}
$$

Proposition 7.2. $\Psi$ is a fibration.

Proof. Let $Y$ be a test space and

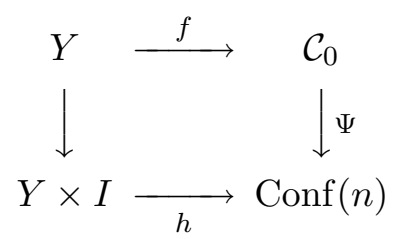

be a commutative test diagram for the homotopy lifting property which defines a fibration. We must show that $h$ lifts to a map $\tilde{h}: Y \times I \rightarrow \mathcal{C}_{0}$ and which commutes with the other maps.

Let us first notice that $h$ can be arbitrarily closely approximated by maps which are smooth in the $I$-direction (for example, by Theorem 2.6 of [Hir76, Chapter 2]). So if we can prove homotopy lifting for such maps then by taking limits of approximation sequences we have proved it for all maps. Let us therefore assume that $h$ is smooth in the $I$-direction.

For clarity of exposition let us also assume that $n=1$ and explain how the proof should be modified for $n>1$ at the end.

The idea behind constructing $\tilde{h}$ will be to find symplectomorphisms $\psi_{y, t}$ : $X \rightarrow X$ such that:

- $\psi_{y, t}$ preserves $C^{\prime}=C_{n+1} \cup C_{n+2}$ and fixes $C_{n+1}$ pointwise,

- $\psi_{y, t}(S) \in \mathcal{C}_{0}$ for any $S \in \mathcal{C}_{0}$ and

- $\Psi\left(\psi_{y, t}(f(y))\right)=h(y, t)$.

The lift $\tilde{h}$ will then be defined by

$$
\tilde{h}(y, t)=\psi_{y, t}(f(y)) .
$$


For example, if $Y=\{y\}$ this is just path-lifting:

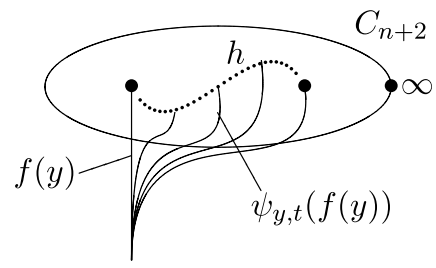

Notice that $\Psi\left(\psi_{y, t}(f(y))\right)=\psi_{y, t}(\Psi(f(y)))$, so it suffices that $\psi_{y, t}$ restricted to $C_{n+2}$ satisfies $\psi_{y, t}(\Psi(f(y)))=h(y, t)$. In fact, we start by constructing the restriction $\bar{\psi}_{y, t}$ of $\psi_{y, t}$ to $C_{n+2}$.

First, pick a continuous family of parametrized Darboux discs $\left\{B_{p} \stackrel{\iota_{p}}{\rightarrow}\right.$ $\left.C_{n+2} \backslash\{\infty\}\right\}$, one centred at each point $p \in C_{n+2} \backslash\{\infty\}$. For each $(y, t) \in Y \times I$ let $X(y, t)=\frac{\partial h(y, t)}{\partial t}$ and let $v(y, t)$ be the pullback of this vector to $B_{h(y, t)}$ along $\iota_{h(y, t)}$. Extend $v$ to a constant vector field on $B_{h(y, t)}$. This is generated by a linear Hamiltonian function which we can multiply by a cut-off function (equal to 1 on a neighbourhood of $\left.0 \in B_{h(y, t)}\right)$ to obtain a Hamiltonian which is compactly supported in the ball. Pulling back this Hamiltonian along $\iota_{h(y, t)}^{-1}$ gives a Hamiltonian $H_{y, t}$ on $C_{n+2}$, supported in a neighbourhood of $h(y, t)$. We call the time $t$ flow of this Hamiltonian $\bar{\psi}_{y, t}$. Notice that $\bar{\psi}_{y, t}(h(y, 0))=h(y, t)$.

Let $N$ be a small tubular neighbourhood of $C_{n+2}, p: N \rightarrow C_{n+2}$ be the projection along the fibres of the pencil $P$ and let $\eta$ be a radial cut-off function on $N$ which equals 1 on a neighbourhood of $C_{n+2}$. The Hamiltonian function $\eta p^{*} H_{y, t}$ generates a time $t$ flow $\psi_{y, t}$ which extends $\bar{\psi}_{y, t}$ in such a way that it fixes $C_{n+1}$ and sends standard configurations to standard configurations. To prove the result when $n>1$ the same construction is used in a Darboux chart near each point of $C_{n+2} \cap S$. This means that now we fix a (continuously varying) choice of $n$ disjoint Darboux discs over each configuration of $n$ distinct points in $C_{n+2} \backslash\{\infty\}$, modifying the proof accordingly.

The space $\operatorname{Conf}(n)$ of configurations of $n$ points in the disc has fundamental group $\mathrm{Br}_{n}$ and since $\mathcal{C}_{0}$ is weakly contractible this group acts freely and transitively on the set of components of the fibre $\mathcal{F}$ of $\Psi$. Moreover, $\operatorname{Conf}(n)$ is a $K\left(\mathrm{Br}_{n}, 1\right)$-space, so the long exact sequence of homotopy groups associated to $\Psi$ implies:

Lemma 7.4. $\mathcal{F} \simeq \pi_{0}(\mathcal{F})$.

We have an action of $\operatorname{Symp}_{c}\left(U^{\prime}\right)$ on the fibre $\mathcal{F}=\Psi^{-1}\left(\left\{\xi_{1}, \ldots, \xi_{n}\right\}\right)$. Let us restrict attention to the orbit $\operatorname{Orb}(E)$ of the standard configuration of exceptional curves $C_{1}, \ldots, C_{n}$.

Lemma 7.5. This consists of a union of connected components of $\mathcal{F}$. 
Proof. If $S_{0} \in \operatorname{Orb}(E) \subset \mathcal{F}$ and $S_{1} \in \mathcal{F}$ lie in the same connected component of $\mathcal{F}$ then they are isotopic through standard configurations; let $S_{t}$ denote such an isotopy. Consider the isotopy $T_{t}=S_{t} \cup C^{\prime}$ of configurations of symplectic spheres. By the assumption that $S_{t}$ is a standard configuration for each $t \in[0,1]$, this isotopy extends to an isotopy of neighbourhoods $\nu_{t}$ of $T_{t}$. We may also assume that this isotopy fixes a neighbourhood of $D=C_{n+2} \cup P_{\infty}$. The fact that $H^{2}\left(X, T_{t} ; \mathbb{R}\right)=0$ implies that this isotopy extends to a global symplectic isotopy of $X$ (by Banyaga's symplectic isotopy extension theorem). Hence there is a symplectomorphism of $X$ compactly supported on the complement of $C^{\prime}$ (i.e., an element of $\operatorname{Symp}_{\mathrm{c}}\left(U^{\prime}\right)$ ) which sends $S_{0}$ to $S_{1}$, so $\operatorname{Orb}(E)$ consists of a union of connected components of $\mathcal{F}$.

Lemma 7.6. The stabilizer $\operatorname{Stab}(E)$ is weakly contractible.

Proof. Arguing as in Lemma 5.2, we see that this is equivalent to showing $\operatorname{Symp}_{\mathrm{c}}(U)$ is weakly contractible. But we have already observed that $\operatorname{Symp}_{\mathrm{c}}(U) \simeq \operatorname{Symp}_{\mathrm{c}}\left(\mathbb{C}^{*} \times \mathbb{C}\right) \simeq \star$.

Banyaga's theorem gives a fibration:

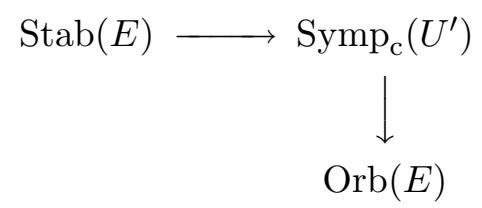

Weak contractibility of $\operatorname{Stab}(E)$ and of the components of $\operatorname{Orb}(E)$ implies that $\pi_{i}\left(\operatorname{Symp}_{\mathrm{c}}\left(U^{\prime}\right)\right)=0$ for $i>0$.

We finally prove that:

Proposition 7.3. $\pi_{0}\left(\operatorname{Symp}_{\mathrm{c}}\left(U^{\prime}\right)\right)$ injects homomorphically into the braid group $\mathrm{Br}_{n}$.

Proof. The symplectomorphisms $\psi_{y, t}$ used to construct lifts in the proof of the fibration property of $\Psi$ are supported in some arbitrarily small neighbourhood of $C_{n+2}$. The action of $\operatorname{Symp}_{c}\left(U^{\prime}\right)$ is by definition supported on the complement of a neighbourhood of $C_{n+2}$. This implies that the action of $\pi_{0}\left(\operatorname{Symp}_{\mathrm{c}}\left(U^{\prime}\right)\right)$ on $\pi_{0}(\mathcal{F})$ commutes with the action of $\mathrm{Br}_{n}$ coming from the fibration $\Psi$. When restricted to the components of $\operatorname{Orb}(E) \subset \mathcal{F}$ we have seen that the $\pi_{0}\left(\operatorname{Symp}_{\mathrm{c}}\left(U^{\prime}\right)\right)$ action is free. The $\mathrm{Br}_{n}$ action is free on the whole of $\pi_{0}(\mathcal{F})$. The claim that $\pi_{0}\left(\operatorname{Symp}_{\mathrm{c}}\left(U^{\prime}\right)\right)$ injects into the braid group now follows from the following elementary lemma:

Lemma 7.7. Let $G$ and $H$ be groups acting on a set $A$. Suppose $H$ acts freely and transitively on $A$ and that $G$ acts freely on an orbit $\operatorname{Orb}_{G}(a)$ for some $a \in A$. In particular, for each $b \in A$ there exists a unique $h_{b}$ such that 
$h_{b}(a)=b$. Define $a$ map

$$
\begin{aligned}
& f: G \rightarrow H, \\
& f(\sigma)=h_{\sigma(a)}^{-1} .
\end{aligned}
$$

This map is injective. If the actions commute then it is a homomorphism.

\section{Appendix A. Structures making a configuration holomorphic}

Let $S=\bigcup_{i=1}^{n} S_{i}$ be a union of embedded symplectic two-spheres in a symplectic four-manifold $X$. Suppose that the various components intersect transversely and that there are no triple intersections. Suppose further that there is an $\omega$-compatible $J$ for which all the components $S_{i}$ are $J$-holomorphic. Let $\mathcal{H}(S)$ denote the space of $\omega$-compatible almost complex structures $J$ for which all components $S_{i}$ are simultaneously $J$-holomorphic. We now provide a proof of Lemma 5.5 from Section 5.2.2.

Lemma 28. If all the intersection between components of $S$ are symplectically orthogonal then the space $\mathcal{H}(S)$ is weakly contractible.

Proof. Let $\mathcal{J}\left(S_{i}\right)$ denote the (contractible) space of $\left.\omega\right|_{S_{i}}$-compatible almost complex structures on $S_{i}$ and $\mathcal{J}(S)$ denote the product $\prod_{i=1}^{n} \mathcal{J}\left(S_{i}\right)$. The space $\mathcal{H}$ maps to $\mathcal{J}(S)$ by restriction. We want to show this is a homotopy equivalence.

We proceed in stages. First define $\left.\mathcal{J}\right|_{S_{i}}$ to be the space of $\omega$-compatible almost complex structures on $\left.T X\right|_{S_{i}}$ and $\left.\mathcal{J}\right|_{S}$ to be the product $\left.\prod_{i=1}^{n} \mathcal{J}\right|_{S_{i}}$. The restriction map $\mathcal{H}(S) \rightarrow \mathcal{J}(S)$ factors through restriction maps:

$$
\left.\mathcal{H}(S) \rightarrow \mathcal{J}\right|_{S} \rightarrow \mathcal{J}(S)
$$

We first analyse the map $\left.\mathcal{J}\right|_{S} \rightarrow \mathcal{J}(S)$.

Lemma A.1. $\left.\mathcal{J}\right|_{S} \rightarrow \mathcal{J}(S)$ is a fibration.

Proof. We illustrate the proof by proving path-lifting. Let $\nu_{i}$ denote the normal bundle to $S_{i}$, identified canonically as a subbundle of $\left.T X\right|_{S_{i}}$ (the $\omega$-orthogonal complement to $\left.T S_{i}\right)$. Fix an $\left.\omega\right|_{\nu_{i}}$-compatible almost complex structure $k_{i}$ on $\nu_{i}$. If

$$
\gamma=\left(j_{1}(\cdot), \ldots, j_{n}(\cdot)\right): I \rightarrow \mathcal{J}(S)=\prod_{i=1}^{n} \mathcal{J}\left(S_{i}\right)
$$

is a path of complex structures on $S=S_{1} \cup \cdots \cup S_{n}$ then $j_{i} \oplus k_{i}$ is an $\omega$-compatible almost complex structure on $\left.T X\right|_{S_{i}}$. Unfortunately, there is no guarantee that $j_{a}(t) \oplus k_{a}=k_{b} \oplus j_{b}(t)$ at the intersection point $x \in$ $S_{a} \cap S_{b}$. To rectify this, fix a Darboux ball $\iota:\left(B, \omega_{0}\right) \rightarrow(X, \omega)$ centred at $x$ for which $\left(S_{a}\right)$ is identified under $\iota$ with $\Pi_{a}=B \cap \mathbb{R}^{2} \times\{0\}$ and $S_{b}$ with 
$\Pi_{b}=B \cap\{0\} \times \mathbb{R}^{2}$. Let $\eta$ be a radial cut-off function on $B$ which is equal to 1 on a neighbourhood of 0 and equal to 0 outside a small ball.

We now modify $k_{a}$ and $k_{b}$ so that they agree with $j_{b}(t)$ and $j_{a}(t)$ at $x$. Let $c$ stands for either $a$ or $b$. Let $g_{\mathrm{c}}$ be the metric on the normal bundle $\nu \Pi_{\mathrm{c}}$ to $\Pi_{\mathrm{c}}$ corresponding to the pullback $\iota^{*} k_{\mathrm{c}}$. Let $j(t)$ be the constant complex structure on $B$ such that $\iota_{*} j(t)=j_{a}(t) \oplus j_{b}(t)$ at $x$. This $j(t)$ defines a metric $\tilde{g}_{\mathrm{c}}(t)$ on the normal bundle to $\Pi_{\mathrm{c}}$. Since the space of metrics is convex, define a metric

$$
g_{\mathrm{c}}^{\prime}(t)=(1-\eta) g_{\mathrm{c}}+\eta \tilde{g}_{\mathrm{c}}(t) .
$$

This in turn defines an endomorphism $A_{\mathrm{c}}(t)$ of $\nu \Pi_{\mathrm{c}}$ via $g_{\mathrm{c}}^{\prime}(t)=\omega_{0}\left(A_{\mathrm{c}}(t) \cdot, \cdot\right)$. By the usual trick, $\left(A_{\mathrm{c}}(t) A_{\mathrm{c}}^{\dagger}(t)\right)^{-1 / 2} A_{\mathrm{c}}(t)$ defines an $\omega_{0}$-compatible almost complex structure on $\nu \Pi_{\mathrm{c}}$. Push this forward along $\iota$. The new almost complex structures $k_{a}^{\prime}(t)$ and $k_{b}^{\prime}(t)$ agree with $k_{a}$ and $k_{b}$ outside the Darboux ball but now $k_{a}^{\prime}(t)=j_{b}(t)$ and $k_{b}^{\prime}(t)=j_{a}(t)$ at $x$.

Do this at every intersection point to get a one-parameter family $k_{i}(t)$ of almost complex structures on $\nu_{i}$. These give almost complex structures $j_{i}(t) \oplus k_{i}(t)$ on $\left.T X\right|_{S_{i}}$ such that $j_{a}(t) \oplus k_{a}(t)=k_{b}(t) \oplus j_{b}(t)$ at any intersection point $x \in S_{a} \cap S_{b}$ and hence we obtain a well-defined lift of $\gamma$ to a path in $\left.\mathcal{J}\right|_{S}$.

Lemma A.2. The fibre of $\left.\mathcal{J}\right|_{S} \rightarrow \mathcal{J}(S)$ is contractible.

Proof. The fibre is the space of almost complex structures on $\left.T X\right|_{S}$ which are fixed along the tangent directions to each $S_{i}$. For each $S_{a}$, let $\mathcal{G}\left(S_{a}\right)$ denote the group of symplectic gauge transformations of $\nu_{a}$ (the normal bundle to $S_{a}$ ) which equal the identity at intersection points $x \in S_{a} \cap S_{b}$. This acts transitively on the space of $\left.\omega\right|_{\nu_{a}}$-compatible almost complex structures which agree with $j_{a} \oplus j_{b}$ at $x \in S_{a} \cap S_{b}$. Hence $\mathcal{G}(S)=\prod_{i=1}^{n} \mathcal{G}\left(S_{i}\right)$ acts transitively on the fibre of $\left.\mathcal{J}\right|_{S} \rightarrow \mathcal{J}(S)$, making it into a homogeneous space $\mathcal{G}(S) / \operatorname{Stab}(J)$. Since the inclusion $U(1) \rightarrow \mathrm{Sp}(2)$ is a homotopy equivalence, $\mathcal{G}(S)$ is homotopy equivalent to the group of unitary gauge transformations, $\mathcal{G}_{u}(S)$, which is also the stabilizer of any given almost complex structure on the normal bundle. The homogeneous space $\mathcal{G}(S) / \mathcal{G}_{u}(S)$ is contractible.

This shows that the space $\left.\mathcal{J}\right|_{S}$ is weakly contractible, as it is the total space of a fibration over a contractible space with contractible fibres. We now analyse the restriction map $\left.\mathcal{H}(S) \rightarrow \mathcal{J}\right|_{S}$.

Lemma A.3. $\left.\mathcal{H}(S) \rightarrow \mathcal{J}\right|_{S}$ is a fibration.

Proof. By the symplectic neighbourhood theorem, each component of $S_{i}$ has a neighbourhood $M_{i}$ isomorphic to a neighbourhood $\nu_{i}$ of the zero section in its normal bundle via an isomorphism $\phi_{i}: \nu_{i} \rightarrow M_{i}$. Since the various components intersect symplectically orthogonally, these identifications can be chosen compatibly in the sense that $S_{j} \cap M_{i}$ is identified with a normal 
fibre in $\nu_{i}$. Let $\gamma(t)=\left(J_{1}(t), \ldots, J_{n}(t)\right)$ be a path in $\left.\mathcal{J}\right|_{S}$. Each $\phi_{i}^{*} J_{i}(t)$ can be canonically extended to an $\omega$-compatible almost complex structure on $\nu_{i}: J_{i}(t)$ automatically defines an almost complex structure on the normal bundle to $S_{i}$, and the horizontal spaces $\omega$-orthogonal to the normal fibres give a connection which allows one to lift $\left.J_{i}(t)\right|_{T S_{i}}$ to an almost complex structure on the total space of $\nu_{i}$.

This does not quite work at the intersection points where one must fix a Darboux chart and implant a local model. Suppose the two intersecting components are sent to the $\mathbb{R}^{2} \times\{0\}$ and $\{0\} \times \mathbb{R}^{2}$ planes in the Darboux chart and the almost complex structure $J(p, q)$ is specified along these planes (i.e., for points $(p, 0)$ and $(0, q))$. Then, working with associated metrics, one can interpolate linearly as follows. Let $S^{1}(r) \times\{0\}$ and $\{0\} \times S^{1}(r)$ denote the unit circles (for the standard Euclidean metric) of the coordinate planes. For any point $x$ of $\mathbb{R}^{4}$ there exists an $r$ and a unique line segment connecting $S^{1}(r) \times\{0\}$ to $\{0\} \times S^{1}(r)$ containing $x$. The analogous picture in $\mathbb{R}^{2}=\mathbb{R} \times \mathbb{R}$ is familiar, where the dots lie on the unit circles:

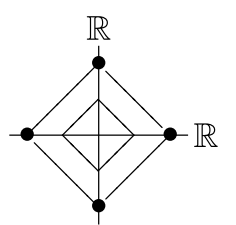

Use the linear coordinate of this line to interpolate the associated metrics. Modifying this near the boundary of the Darboux ball to agree with the $J$ constructed from connections, we obtain an $\omega$-compatible almost complex structure $\tilde{\gamma}(t)$ on a neighbourhood of $S$.

Fix an arbitrary $\omega$-compatible almost complex structure $K$ on $X$. Interpolating associated metrics using a cut-off function in a neighbourhood of $S$, one can extend $\tilde{\gamma}(t)$ over $X$ so that it agrees with $K$ outside a neighbourhood of $S$. This gives a lift of the path $\gamma$. The proof of the covering homotopy property is similar but cumbersome.

Lemma A.4. The fibre of $\left.\mathcal{H}(S) \rightarrow \mathcal{J}\right|_{S}$ is contractible.

Proof. This is standard. The fibre $\mathcal{F}$ consists of $\omega$-compatible almost complex structures making $S$ holomorphic which agree with a fixed almost complex structure $J$ along $S$. We define a deformation retraction of $\mathcal{F}$ to $\{J\}$ : for $J^{\prime} \in \mathcal{F}$, define the metric $g_{J^{\prime}}=\omega\left(\cdot, J^{\prime} \cdot\right)$. The path $g_{J^{\prime}}(t)=t g_{J}+(1-t) g_{J^{\prime}}$ of metrics defines a path of almost complex structures connecting $J^{\prime}$ and $J$ in the usual way.

This shows that $\mathcal{H}(S)$ is weakly contractible, since it is the total space of a fibration over a weakly contractible space $\left.\mathcal{J}\right|_{S}$ with contractible fibres. 


\section{References}

[Abr98] M. Abreu, Topology of symplectomorphism groups of $S^{2} \times S^{2}$, Invent. Math. 131 (1998), 1-23.

[BC01] P. Biran and K. Cieliebak, Symplectic topology on subcritical manifolds, Comment. Math. Helv. 76(4) (2001) 712-753.

[BM06] R. Bell and D. Margalit. Braid groups and the co-Hopfian property, J. Algebra 303 (2006) 275-294.

[EE67] C. J. Earle and J. Eells, The diffeomorphism group of a compact Riemann surface, Bull. Amer. Math. Soc. 73 (1967) 557-559.

[GH94] P. Griffiths and J. Harris. Principles of algebraic geometry, Wiley Interscience, Wiley Classics Library edition, New York, 1994.

[Gom95] R. Gompf, A new construction of symplectic manifolds, Ann. Math. 142(3) (1995) 527-595.

[Gro85] M. Gromov, Pseudoholomorphic curves in symplectic manifolds, Invent. Math. 82 (1985) 307-347.

[Hin03] R. Hind, Stein fillings of lens spaces, Commun. Contemp. Math. 5 (2003) 967982.

[Hir76] M. Hirsch, Differential topology, Graduate Texts in Math., 33, Springer, Berlin, 1976.

[KS01] M. Khovanov and P. Seidel, Quivers, Floer cohomology and braid group actions, J. Amer. Math. Soc. 15(1) (2001) 203-271.

[LP04] F. Lalonde and M. Pinsonnault, The topology of the space of symplectic balls in rational 4-manifolds, Duke Math. J. 122(2) (2004) 347-397.

[MS04] D. McDuff and D. Salamon, J-holomorphic curves and symplectic topology, Colloquium Publications, 52, American Mathematical Society, Providence, RI, 2004.

[MS05] D. McDuff and D. Salamon, Introduction to symplectic topology, 2nd ed., Oxford Math. Monographs, OUP, 2005.

[Pin08] M. Pinsonnault, Maximal tori in the Hamiltonian groups of 4-dimensional symplectic manifolds, J. Mod. Dynam. 2(3) (2008) 431-455.

[Pin] M. Pinsonnault, private communication.

[Sei98] P. Seidel, Symplectic automorphisms of $T^{*} S^{2}$, arXiv preprint, arXiv:math/9803084v1, 1998.

[Sei08] P. Seidel, Lectures on four-dimensional Dehn twists, in Symplectic 4-Manifolds and Algebraic Surfaces, Lecture Notes Math., 1938, Springer, Berlin, 2008, 231268 .

[SS05] P. Seidel and I. Smith, The symplectic topology of Ramanujam's surface, Comment. Math. Helv. 80(4) (2005) 859-881.

[Wen08] C. Wendl, Strongly fillable contact manifolds and J-holomorphic foliations, arXiv:0806.3193, 2008.

Department of Mathematics

ETH ZürICH, RÄMISTRAsse 101, 8092 ZürICH, SwITZERland

E-mail address: jonathan.evans@math.ethz.ch

Received 09/30/2009, accepted 07/13/2010 
During this work I was funded by a Faulkes Studentship in Geometry. This work formed part of my $\mathrm{PhD}$ thesis at the University of Cambridge and it gives me pleasure to acknowledge the many stimulating conversations with my Ph.D. supervisor, Ivan Smith, which have led to this paper. I would also like to thank Jack Waldron, Mark McLean, Chris Wendl, Jarek Kȩdra, Gabriel Paternain, Dusa McDuff and the paper's referee for useful comments, encouragement and corrections. Particular thanks go to Jack Button for his comments and suggestions about the co-Hopfian properties of groups and to Chris Wendl for explaining steps in his paper [Wen08]. The ideas and arguments in this paper are heavily inspired by sections of Seidel's marvellous survey [Sei08] and Abreu's beautiful paper [Abr98]. Since preparing this paper, the author has learned that the results on monotone symplectic Del Pezzo surfaces have also been obtained independently by Martin Pinsonnault $[\mathbf{P i n}]$. 